\title{
Fully Implicit Multidimensional Hybrid Upwind Scheme for Coupled Flow and Transport
}

\author{
François P. Hamon ${ }^{\mathrm{a}, *}$, Bradley T. Mallison ${ }^{\mathrm{b}}$ \\ ${ }^{a}$ Center for Computational Sciences and Engineering, Lawrence Berkeley National Laboratory, Berkeley, USA \\ ${ }^{b}$ Chevron ETC, 6001 Bollinger Canyon Rd, San Ramon, USA
}

\begin{abstract}
Robust and accurate fully implicit finite-volume schemes applied to Darcy-scale multiphase flow and transport in porous media are highly desirable. Recently, a smooth approximation of the saturationdependent flux coefficients based on Implicit Hybrid Upwinding (IHU) has been proposed to improve the nonlinear convergence in fully implicit simulations with buoyancy. Here, we design a truly multidimensional extension of this approach that retains the simplicity and robustness of IHU while reducing the sensitivity of the results to the orientation of the computational (Cartesian) grid. This is achieved with the introduction of an adaptive, local coupling between the fluxes that takes the flow pattern into account. We analyze the mathematical properties of the proposed methodology to show that the scheme is monotone in the presence of competing viscous and buoyancy forces and yields saturations remaining between physical bounds. Finally, we demonstrate the efficiency and accuracy of the scheme on challenging two-dimensional two-phase examples with buoyancy, with an emphasis on the reduction of the grid orientation effect.
\end{abstract}

Keywords: Porous media, Two-phase flow and transport, Implicit finite-volume schemes, Grid-orientation effect, Truly multidimensional schemes, Upwinding

\section{Introduction}

The numerical simulation of subsurface flow requires the design of accurate and robust discretization schemes for the highly nonlinear partial differential equations (PDEs) governing coupled flow and transport in porous media. The high heterogeneity characterizing geological porous media constitutes a challenge for the computational models used in practice. In realistic models, the rock properties (permeability and porosity) can vary by several orders of magnitude, which results in a vast range of flow velocities and time scales in the hyperbolic transport of species. High localized flow velocities impose severe restrictions on the time step size for explicit time integration methods. Therefore, a fully implicit, backward-Euler method guaranteeing unconditional stability is often preferred in the case of strongly nonlinear problems with high heterogeneity.

However, constructing accurate and efficient fully implicit schemes for subsurface flow simulation is a challenging task. In particular, the nonlinearity of the saturation-dependent coefficients present in the flux terms makes the application of high-resolution schemes challenging despite some encouraging recent efforts (Mykkeltvedt et al., 2017; Arbogast et al., 2019). Therefore, these coefficients are often approximated with robust first-order upwind schemes such as the commonly used Phase-Potential Upwinding (PPU, Peaceman, 1977; Aziz and Settari, 1979, Sammon, 1988, Brenier and Jaffré, 1991). Despite limitations with respect to resolution, first-order schemes are preferred in practice because they can accommodate large time steps when combined with Newton or quasi-Newton nonlinear iterations. But, even limited to the family of first-order schemes, the choice of an upwinding strategy has a significant impact on the accuracy of the flow predictions and on the computational cost of the numerical scheme.

Previous authors have shown that nonlinear convergence issues can result from the approximation of the interfacial phase flux (Wang and Tchelepi, 2013, Li and Tchelepi, 2015) and can consequently

\footnotetext{
${ }^{*}$ Corresponding author, now with TOTAL Exploration \& Production USA
} 


\section{Hamon and Mallison}

undermine the performance of the scheme since the fully implicit discretization requires solving large nonlinear systems at each time step. Specifically, when the approximation is based on PPU, convergence difficulties may arise when a small change in the primary variables - pressure and saturation - causes an abrupt change from cocurrent flow to countercurrent flow, and vice versa. This issue, referred to as the "flip-flopping" of the phase fluxes, was recently addressed with Implicit Hybrid Upwinding (IHU) in Lee et al. (2015); Lee and Efendiev (2016, 2018); Hamon and Tchelepi (2016); Hamon et al. (2016, 2018); Moncorgé et al. (2019). The IHU strategy is based on a separate evaluation of the viscous, buoyancy, and capillary parts to achieve a differentiable flux in the highly nonlinear transport problem. This reduces the flip-flopping of the phase fluxes and significantly improves the nonlinear convergence of Newton's method while producing a similar accuracy as that of the standard PPU scheme.

The computational challenges are even greater in multiphase flows where the displacing phase has greater mobility than the displaced phase. Both the standard PPU and IHU upwinding procedures have limited accuracy for adverse mobility ratios because the saturation-dependent coefficients are approximated dimension-by-dimension. That is, the construction of the flux at an interface is entirely based on the saturation information contained in the two adjacent control volumes. A well-known issue resulting from this approach is the high sensitivity of the flow pattern to the orientation of the computational grid. In practice, these dimension-by-dimension schemes are therefore inaccurate when the flow is not aligned with the orientation of the computational grid. This unphysical grid orientation effect has been studied extensively for porous media problems (Yanosik and McCracken, 1979, Pruess and Bodvarsson, 1983 Shubin and Bell, 1984, Brand et al., 1991). So-called multidimensional schemes taking into account the saturation information in an extended stencil around each interface have been designed to overcome this limitation of two-point upwinding. Following the work of Colella (1990); Koren (1991); Roe and Sidilkover (1992); LeVeque (1997); Berger et al. (2003) in computational fluid dynamics, the multidimensional schemes tailored for subsurface flow simulation include Chen et al. (1993); Arbogast and Huang (2006); Hurtado et al. (2007); Lamine and Edwards (2010, 2013, 2015); Eymard et al. (2012).

But, few of these schemes can be efficiently combined with a fully implicit discretization targeting highly nonlinear problems with competing viscous and buoyancy forces. In Kozdon et al. (2011a b); Keilegavlen et al. (2012), the authors design a multidimensional scheme based on PPU that remains robust in fully implicit simulations of coupled multiphase flow and transport, and that is provably monotone in the presence of buoyancy. In this paper, we build on their approach to construct an efficient and accurate multidimensional IHU scheme. As in Kozdon et al. (2011a), we neglect capillary forces and focus on the mixed elliptic-hyperbolic incompressible two-phase flow and transport problem with viscous and buoyancy forces discretized on two-dimensional Cartesian grids. We propose a scheme that reduces the sensitivity of the results to the orientation of the computational grid while preserving a robust behavior of the Newton solver in the presence of competing viscous and buoyancy forces.

This is achieved by adapting the methodology previously used to construct the multidimensional PPU scheme. Specifically, to compute the viscous numerical flux, we introduce a local coupling between the phase fluxes belonging to the same interaction region of the dual grid. The buoyancy flux is computed separately and is upwinded based on fixed density differences. Importantly, this approach yields a numerical flux that is a monotone function of the saturations in the presence of viscous and buoyancy forces. We also prove that the saturation solution to the proposed fully implicit scheme remains between physical bounds, zero and one. Using numerical examples, we demonstrate that the proposed scheme is significantly less sensitive to the orientation of the grid than the two-point PPU and IHU approaches. We also illustrate the robustness of the multidimensional IHU by applying the scheme to challenging nonlinear test cases with large time steps.

We first describe the mathematical model describing coupled multiphase flow and transport with viscous and buoyancy forces in Section 2. Then, in Section 3, we proceed with the description of the fully implicit finite-volume scheme and we define the dual grid as the union of interaction regions. These interaction regions are used to introduce the truly multidimensional IHU in Section 4 and to study its mathematical properties in Section 5 . Finally, using the nonlinear solver described in Section 6, we demonstrate the accuracy and robustness of the multidimensional scheme using numerical examples with buoyancy in Section 7 


\section{Governing equations}

In this work, we consider two immiscible and incompressible fluid phases flowing in an incompressible porous medium. Mass conservation for phase $\ell$ is expressed as

$$
\phi \frac{\partial S_{\ell}}{\partial t}+\nabla \cdot \mathbf{u}_{\ell}=q_{\ell} \quad \forall \mathbf{x} \in \Omega \subset \mathbb{R}^{2}, \quad \forall \ell \in\{n w, w\},
$$

where $\phi(\mathbf{x})$ is the porosity of the medium and $t$ is time. The source/sink term is denoted by $q_{\ell}$, with the convention that $q_{\ell}>0$ for injection, and $q_{\ell}<0$ for production. The saturation $S_{\ell}(\mathbf{x}, t)$ represents the fraction of the pore volume occupied by phase $\ell$, with the constraint that the sum of saturations is equal to one,

$$
\sum_{\ell} S_{\ell}=1
$$

Using the multiphase extension of Darcy's law, the phase velocity $\mathbf{u}_{\ell}$ is written as a function of the phase potential gradient $\nabla \Phi_{\ell}$ as

$$
\mathbf{u}_{\ell}:=-k \lambda_{\ell} \nabla \Phi_{\ell}=-k \lambda_{\ell}\left(\nabla p-\mathbf{g}_{\ell}\right) \quad \text { with } \mathbf{g}_{\ell}=\rho_{\ell} g \nabla z, \quad \forall \ell \in\{n w, w\},
$$

where capillary forces are neglected. In (3), $p(\mathbf{x}, t)$ is the pressure, $k(\mathbf{x})$ is the scalar rock permeability, $\lambda_{\ell}\left(S_{n w}, S_{w}\right)=k_{r \ell}\left(S_{n w}, S_{w}\right) / \mu_{\ell}$ is the phase mobility - defined as the phase relative permeability divided by the phase viscosity,$- \rho_{\ell}$ is the phase density, $g$ is the gravitational acceleration, and $z$ is the depth. We require that the mobilities be monotone functions of the saturations, which is realistic and holds for two-phase relative permeability models such as the Corey model (Corey, 1954).

Assumption 1. (Phase mobilities). For $\ell \in\{n w, w\}$, the mobility of phase $\ell$ is positive, and a differentiable function of the saturations. Furthermore, the mobility of phase $\ell$ is increasing with respect to its saturation and decreasing with respect to the saturation of the other phases:

$$
\frac{\partial \lambda_{\ell}}{\partial S_{\ell}} \geq 0 \quad \text { and } \quad \frac{\partial \lambda_{\ell}}{\partial S_{m}} \leq 0 \quad \forall m \neq \ell .
$$

The system of mixed elliptic-hyperbolic governing PDEs can be written in two equivalent forms, where the linearly independent primary variables are the pressure, $p$, and the wetting-phase saturation, denoted by $S=S_{w}$ for simplicity. In the remainder of this paper, we use the saturation constraint (2) to write the saturation-dependent properties as a function of $S$ only. The first form of the governing PDEs is obtained by inserting the expression of the phase velocities given by Darcy's law (3) into the mass conservation equations (1),

$$
\phi \frac{\partial S_{\ell}}{\partial t}-\nabla \cdot\left(k \lambda_{\ell}\left(\nabla p-\mathbf{g}_{\ell}\right)\right)=q_{\ell} \quad \forall \ell \in\{n w, w\} .
$$

The second form of the governing PDEs is split into a flow problem and a transport problem. The flow problem is obtained by summing (1) over all phases and using the saturation constraint (2) to obtain:

$$
\nabla \cdot \boldsymbol{u}_{T}(\boldsymbol{x}, p, S)=\sum_{\ell} q_{\ell}=q_{T},
$$

where the total velocity, $\mathbf{u}_{T}$, is defined as the sum of the phase velocities:

$$
\mathbf{u}_{T}(\mathbf{x}, p, S):=\sum_{\ell} \mathbf{u}_{\ell}(\mathbf{x}, p, S)=-k \lambda_{T} \nabla p+k \sum_{\ell} \lambda_{\ell} \mathbf{g}_{\ell} .
$$

We assume that the following assumption on the total mobility holds:

Assumption 2. (Total mobility). The total mobility $\lambda_{T}=\sum_{\ell} \lambda_{\ell}$ is bounded away from zero:

$$
0<\epsilon \leq \lambda_{T}(S) \leq \chi \quad \forall S \in[0,1] .
$$




\section{Hamon and Mallison}

Equation (6), referred to as the pressure equation, is parabolic when compressibility is taken into account (Trangenstein and Bell, 1989a b), and elliptic in the incompressible case that we will exclusively consider. The flow problem governs the evolution of the pressure variables as a function of space and time. It is coupled to the highly nonlinear transport of species, derived next. Equation (7) is used to express the pressure gradient as a function of $\mathbf{u}_{T}$ and the weights $\mathbf{g}_{\ell}(\ell \in\{n w, w\})$, in order to eliminate the pressure variable from $(3)$. We obtain

$$
\mathbf{u}_{\ell}(\mathbf{x}, p, S)=\frac{\lambda_{\ell}}{\lambda_{T}} \mathbf{u}_{T}(\mathbf{x}, p, S)+k \sum_{m} \frac{\lambda_{m} \lambda_{\ell}}{\lambda_{T}}\left(\mathbf{g}_{\ell}-\mathbf{g}_{m}\right)
$$

This allows us to rewrite the system of PDEs in the following fractional flow formulation which governs the hyperbolic transport of species as

$$
\phi \frac{\partial S_{\ell}}{\partial t}+\nabla \cdot\left(\frac{\lambda_{\ell}}{\lambda_{T}} \mathbf{u}_{T}(\mathbf{x}, p, S)+k \sum_{m} \frac{\lambda_{m} \lambda_{\ell}}{\lambda_{T}}\left(\mathbf{g}_{\ell}-\mathbf{g}_{m}\right)\right)=q_{\ell} \quad \forall \ell \in\{n w, w\} .
$$

The system composed of (6) and 10 contains a redundant equation. Although it is possible to solve both equations in (10) by relaxing the saturation constraint, we enforce the saturation constraint and solve (6) along with (10) only for $\ell=w$. We highlight that in the two equivalent systems of governing PDEs of (5), or (6) and (10), the flow is tightly coupled to the highly nonlinear transport of species through the mobility terms. Next, we employ a fully implicit finite-volume scheme to discretize the system of governing PDEs, with an emphasis on the truly multidimensional computation of the numerical flux aiming at reducing grid orientation effects. As explained below, the proposed methodology is based on the fractional flow formulation and builds on the Implicit Hybrid Upwinding approach (Lee et al., 2015) to construct the flux approximation.

\section{Numerical scheme}

\subsection{Integration region framework}

We consider a two-dimensional Cartesian grid consisting of $M$ control volumes discretizing the domain $\Omega$. In the multidimensional scheme proposed in this paper, the flux is computed with an adaptive stencil containing at least two upwind control volumes in the neighborhood of the interface under consideration. The weights given to the upwind volumes are chosen to improve accuracy by accounting for characteristic information while also honoring monotonicity. To define this stencil, we adapt the approach previously used in Kozdon et al. (2011a); Keilegavlen et al. (2012) and use a dual grid made of the union of interaction regions as illustrated in Fig. 1. Control-volume finite element methods (Schneider and Raw, 1986) and multi-point flux finite volume methods (Aavatsmark et al., 1998, Edwards and Rogers, 1998, Aavatsmark, 2002 ) utilize similar definitions of interaction regions and dual grids.

Each control volume in the primal grid is part of four interaction regions. As in Kozdon et al. (2011a), these interaction regions are locally labeled counterclockwise using the superscript $(m)$, with $m \in\{1, \ldots, 4\}$, starting from the bottom left corner of the control volume. In the dual grid, the four vertices of an interior interaction region correspond to the centers of the four control volumes intersected by this interaction region. These four vertices are locally labeled counterclockwise with the subscript $k \in\{1, \ldots, 4\}$, starting from the bottom left vertex of the interaction region. Because we are developing first-order schemes, the primary variables are located at the centers of the control volumes in the primal grid, or equivalently, at the vertices of the interaction regions in the dual grid. We denote by $p_{k}^{(m)}$ (respectively, $S_{k}^{(m)}$ ) the pressure (respectively, the wetting-phase saturation) located at the $k^{\text {th }}$ vertex of interaction region $(m)$. We point out that $p_{3}^{(1)}, p_{4}^{(2)}, p_{1}^{(3)}$, and $p_{2}^{(4)}$ refer to the same degree of freedom viewed from different interaction regions.

The flux computation is performed at the four control volume segments contained in each interaction region and referred to as half interfaces. We write the quantities computed at these half interfaces with an overline to distinguish them from the control-volume centered quantities. The four half interfaces are also locally numbered counterclockwise with the subscript $k+1 / 2 \in\{1+1 / 2, \ldots, 4+1 / 2\}$. In interaction 


\section{Fully Implicit Multidimensional Hybrid Upwind Scheme}

region $(m)$, we denote by $\bar{F}_{\ell, k+1 / 2}^{(m)}$ the approximation of the integral of the velocity of phase $\ell$ at the half interface $\Gamma_{k+1 / 2}^{(m)}$ between vertex $k$ and $k+1$, defined as

$$
\bar{F}_{\ell, k+1 / 2}^{(m)} \approx \int_{\Gamma_{k+1 / 2}^{(m)}} \boldsymbol{u}_{\ell}(\boldsymbol{x}, p, S) \cdot \boldsymbol{n}_{k+1 / 2}^{(m)} \mathrm{d} \Gamma_{k+1 / 2}^{(m)},
$$

where $\boldsymbol{n}_{k+1 / 2}^{(m)}$ is the outward normal to $\Gamma_{k+1 / 2}^{(m)}$. In our convention, $\bar{F}_{\ell, k+1 / 2}^{(m)} \geq 0$ means that the positive flux for phase $\ell$ at half interface $\Gamma_{k+1 / 2}^{(m)}$ is from vertex $k$ to vertex $k+1$ in interaction region $(m)$. Similarly, considering the total velocity at half interface $\Gamma_{k+1 / 2}^{(m)}$, which satisfies

$$
\bar{u}_{T, k+1 / 2}^{(m)}=\sum_{\ell} \bar{F}_{\ell, k+1 / 2}^{(m)}
$$

the inequality $\bar{u}_{T, k+1 / 2}^{(m)} \geq 0$ means that the positive total flux at half interface $\Gamma_{k+1 / 2}^{(m)}$ is from vertex $k$ to $k+1$. Note that the subscript $k$ is defined cyclically on $\{1, \ldots, 4\}$, and that, with a slight abuse of notation, we write $k+1$ instead of $\bmod (k, 4)+1$ in the previous discussion. With this convention, $k+1$ indicates a counterclockwise step around an interaction region and $k-1$ indicates a clockwise step.

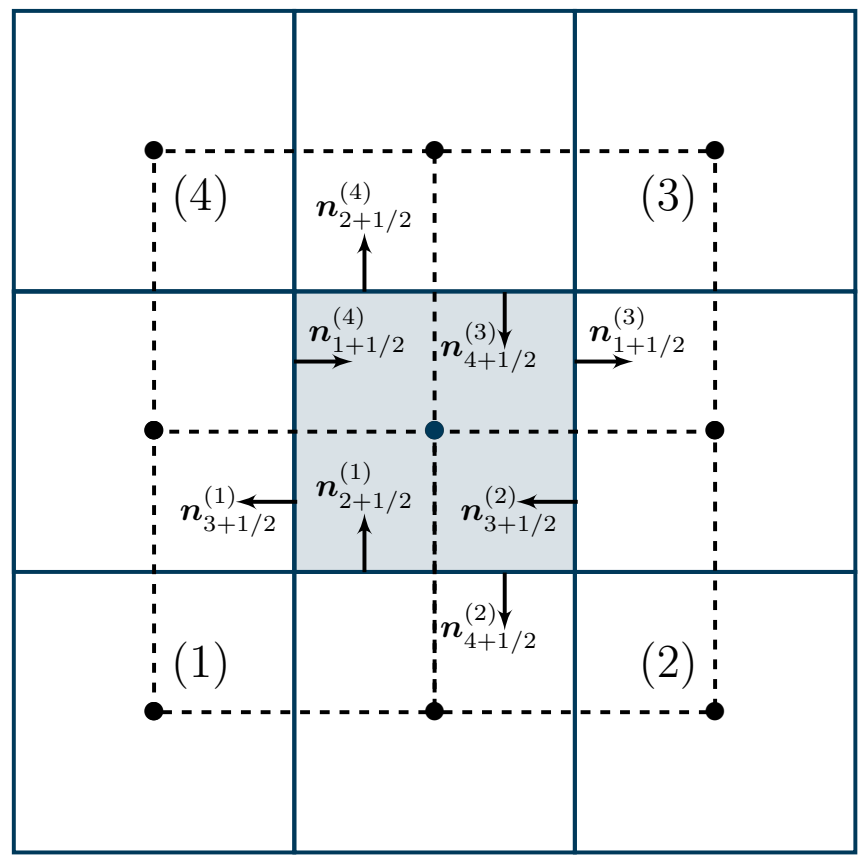

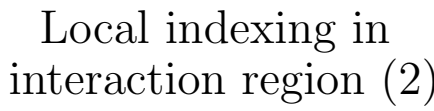

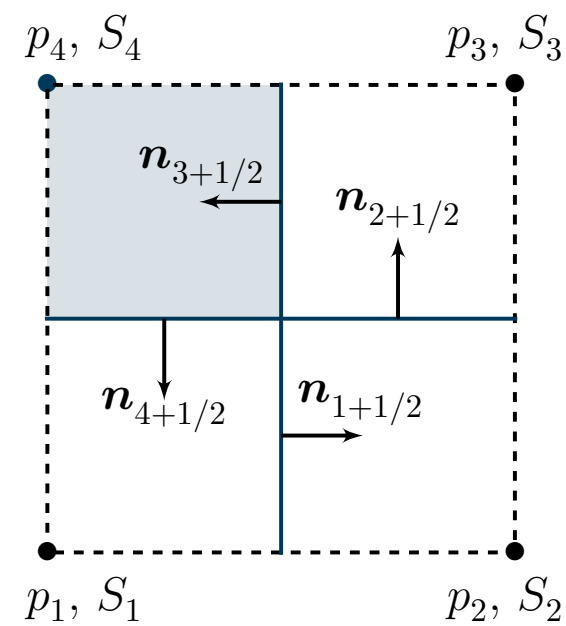

Figure 1: Primal grid (solid blue line) and dual grid (dashed black line). The schematic on the left shows the four interaction regions intersecting the control volume in light blue. The schematic on the right focuses on interaction region (2) located at the bottom right of the control volume in light blue. In this schematic, we omitted the superscript (2) denoting the interaction region index. The dots show the location of the control volume centers, or, equivalently, the vertices of the interaction regions. The arrows show the outward normals to the half interfaces. There are four half interfaces associated with each interaction region and, as a result, there are eight half interfaces associated with a primal control volume.

\subsection{Implicit finite-volume scheme}

We are ready to write the discrete version of the system of governing equations $(6)-(10)$. We consider the control volume in blue in Fig. 1. Viewed from interaction region (2), the pressure and saturation of 


\section{Hamon and Mallison}

phase $\ell$ at the center of this control volume are on the fourth vertex and are denoted by $p_{\ell, 4}^{(2)}$ and $S_{\ell, 4}^{(2)}$. The statement of discrete mass conservation for phase $\ell \in\{1,2\}$ in this control volume reads

$$
V \phi \frac{S_{\ell, 4}^{(2)}-\left(S_{\ell, 4}^{(2)}\right)^{n}}{\Delta t^{n}}+\sum_{k}\left(\bar{F}_{\ell, k+1 / 2}^{(k-2)}-\bar{F}_{\ell, k-1 / 2}^{(k-2)}\right)=V q_{\ell},
$$

where $\Delta t^{n}=t^{n+1}-t^{n}$ is the time step and $V$ is the volume of the control volume. We assume fixed positive injection terms balanced by variable (i.e., pressure-controlled) negative production terms. The flux term can be split to isolate the contribution from each interaction region as

$$
\begin{aligned}
\sum_{k}\left(\bar{F}_{\ell, k+1 / 2}^{(k-2)}-\bar{F}_{\ell, k-1 / 2}^{(k-2)}\right) & =\underbrace{\bar{F}_{\ell, 3+1 / 2}^{(1)}-\bar{F}_{\ell, 2+1 / 2}^{(1)}}_{\begin{array}{c}
\text { interaction } \\
\text { region }(1)
\end{array}}+\underbrace{\bar{F}_{\ell, 4+1 / 2}^{(2)}-\bar{F}_{\ell, 3+1 / 2}^{(2)}}_{\begin{array}{c}
\text { interaction } \\
\text { region (2) }
\end{array}} \\
& +\underbrace{\bar{F}_{\ell, 1+1 / 2}^{(3)}-\bar{F}_{\ell, 4+1 / 2}^{(3)}}_{\begin{array}{c}
\text { interaction } \\
\text { region (3) }
\end{array}}+\underbrace{\bar{F}_{\ell, 2+1 / 2}^{(4)}-\bar{F}_{\ell, 1+1 / 2}^{(4)}}_{\begin{array}{c}
\text { interaction } \\
\text { region (4) }
\end{array}}
\end{aligned}
$$

Summing 13 over all phases and using 12 to form the total velocity results in the discrete pressure equation:

$$
\sum_{k}\left(\bar{u}_{T, k+1 / 2}^{(k-2)}-\bar{u}_{T, k-1 / 2}^{(k-2)}\right)=V q_{T},
$$

where we have used the saturation constraint (2) to cancel the derivatives of saturation on the left-hand side. Finally, we underline the fact that we use a fully implicit discretization, with all the quantities evaluated at the most recent time and iteration level, except the saturation at the previous time in the accumulation term.

\subsection{Flux approximation}

The flux defined in (11) can be decomposed into two terms. The first term is a static transmissibility coefficient depending on the rock properties and the grid geometry in a stencil involving multiple control volumes in the neighborhood of the interface. This term is independent of the saturations and can be computed in a preprocessing step. In this work, we limit the scope of the numerical study to Cartesian grids with scalar permeability and therefore exclusively consider a Two-Point Flux Approximation (TPFA) to compute this transmissibility term. Multi-Point Flux Approximations (MPFA, Aavatsmark et al., 1998; Edwards and Rogers, 1998, Aavatsmark, 2002, Zhou et al., 2011) are required for more general cases as previously shown by Keilegavlen et al. (2012); Lamine and Edwards (2013); Souza et al. (2018). Considering half interface $\Gamma_{k+1 / 2}^{(m)}$ between vertices $k$ and $k+1$, we define the rock-and geometric transmissibility as

$$
\bar{T}_{k+1 / 2}^{(m)}:=\frac{2 l_{k+1 / 2}}{d_{k+1 / 2}\left(\frac{1}{k_{k}}+\frac{1}{k_{k+1}}\right)} .
$$

In $\sqrt{16}, l_{k+1 / 2}$ is the length of half interface $\Gamma_{k+1 / 2}^{(m)}, d_{k+1 / 2}$ is the distance between vertices $k$ and $k+1$, and $k_{k}$ (respectively, $k_{k+1}$ ) denotes the absolute permeability in the control volume centered at vertex $k$ (respectively, vertex $k+1$ ). This choice implies that in two consecutive interaction regions - e.g., (1) and (2) in Fig. 1 - the two half interfaces splitting a full control-volume interface $-\Gamma_{2+1 / 2}^{(1)}$ and $\Gamma_{4+1 / 2}^{(2)}-$ have the same transmissibility - that is, $\bar{T}_{2+1 / 2}^{(1)}=\bar{T}_{4+1 / 2}^{(2)}$. In the ordering of Fig. 1 . we have

$$
\bar{T}_{k-3 / 2}^{(k+1)}=\bar{T}_{k+1 / 2}^{(k+2)}
$$

The second term is a highly nonlinear, non-convex function of the saturations whose computation has a significant impact on the robustness and the accuracy of the numerical scheme. Even though high-resolution schemes have been developed for multiphase flow in porous media Blunt and Rubin, 
1992; Mallison et al., 2005; Lamine and Edwards, 2010; Mykkeltvedt et al., 2017), we focus on commonly used monotone first-order upwinding schemes and will consider higher-resolution extensions in future work. Robust two-point upwinding schemes such as Phase-Potential Upwinding (PPU) and Implicit Hybrid Upwinding (IHU) are relatively simple and inexpensive but suffer from a strong sensitivity to the orientation of the computational grid for flows involving adverse mobility ratios. This leads to inaccurate predictions when the flow is not aligned with the grid, which is often the case in practical simulations.

In this work, we address this issue with a multidimensional evaluation of the saturation-dependent coefficients based on a larger stencil that adapts to the flow direction. Our approach extends the original IHU approach to improve the accuracy of the fully implicit numerical scheme while retaining the robustness of the IHU scheme in the presence of strong buoyancy. The scheme is constructed to reduce the sensitivity of the results to the orientation of the computational grid by introducing a local coupling between the fluxes in each interaction region. A half flux can therefore be a function of all the saturations in the interaction region, leading to a stencil with a maximum of nine points in the discretization of the mobilities. To guarantee that the resulting scheme is well behaved, we require that for a fixed total velocity field, the fluxes satisfy the following monotonicity constraint in interaction region $(m)$ :

$$
\frac{\partial\left(\bar{F}_{\ell, k+1 / 2}^{(m)}-\bar{F}_{\ell, k-1 / 2}^{(m)}\right)}{\partial S_{\ell, j \neq k}^{(m)}} \leq 0 \quad \forall k \in\{1, \ldots, 4\}, \quad \forall \ell \in\{n w, w\} .
$$

It follows from a mass conservation statement written at two consecutive half interfaces $\Gamma_{k-1 / 2}^{(m)}$ and $\Gamma_{k+1 / 2}^{(m)}$ that whenever the monotonicity constraint holds we also have

$$
\frac{\partial\left(\bar{F}_{\ell, k+1 / 2}^{(m)}-\bar{F}_{\ell, k-1 / 2}^{(m)}\right)}{\partial S_{\ell, k}^{(m)}} \geq 0 \quad \forall \ell \in\{n w, w\} .
$$

A similar monotonicity condition is used in Kozdon et al. (2011a); Keilegavlen et al. (2012). In Section 5, we use this property to show that the saturation solution of the fully implicit scheme remains between physical bounds, 0 and 1.

\section{Multidimensional Implicit Hybrid Upwinding}

In the following sections, we consider an interaction region $(m)$ and we detail the discretization of the interfacial quantities in the four half fluxes. To construct the approximation, we follow the Implicit Hybrid Upwinding (IHU) approach (Eymard et al., 1989, Lee et al., 2015, Lee and Efendiev, 2016, 2018. Hamon and Tchelepi, 2016, Hamon et al., 2016, 2018, Moncorgé et al., 2019, Xie and Edwards, 2019) and split the numerical flux at half interface $\Gamma_{k+1 / 2}$ between vertices $k$ and $k+1$ into a viscous part, $\bar{V}_{\ell, k+1 / 2}$, and a buoyancy part, $\bar{G}_{\ell, k+1 / 2}$, as follows

$$
\begin{aligned}
\bar{F}_{\ell, k+1 / 2} & =\bar{V}_{\ell, k+1 / 2}+\bar{G}_{\ell, k+1 / 2} \\
& \approx \int_{\Gamma_{k+1 / 2}} \frac{\lambda_{\ell}}{\lambda_{T}} \mathbf{u}_{T}(\mathbf{x}, p, S) \cdot \boldsymbol{n}_{k+1 / 2} \mathrm{~d} \Gamma_{k+1 / 2} \\
& +\sum_{m} \int_{\Gamma_{k+1 / 2}} k \frac{\lambda_{m} \lambda_{\ell}}{\lambda_{T}}\left(\mathbf{g}_{\ell}-\mathbf{g}_{m}\right) \cdot \boldsymbol{n}_{k+1 / 2} \mathrm{~d} \Gamma_{k+1 / 2},
\end{aligned}
$$

where we omitted the superscript denoting the interaction region. The viscous and buoyancy parts are then evaluated separately, based on physical considerations, to achieve a differentiable flux when the total velocity field is fixed. The proposed discretization of $\bar{V}_{\ell, k+1 / 2}$ and $\bar{G}_{\ell, k+1 / 2}$, explained next, attenuates grid orientation effects when the flow is not aligned with the computational grid, but reduces to the original IHU when the flow is aligned with the computational grid. We show in the next sections that it satisfies the monotonicity property outlined above, and that it preserves a robust nonlinear convergence behavior even in the presence of strong buoyancy forces. 


\section{Hamon and Mallison}

Multiple strategies have been used to reflect the local flow pattern in the construction of multidimensional upwind schemes for porous media flow. In the IMPES and fully implicit multidimensional PPU schemes of Kozdon et al. (2011a b); Keilegavlen et al. (2012), the authors design a flux approximation based on (5) by directly computing weighted averages of mobilities to account for the flow orientation in each interaction region. Instead, in the IMPES fractional-flow based schemes of Lamine and Edwards (2010, 2013, 2015); Edwards (2011), the flow orientation is exploited in the computation of weighted averages of saturations - which are then used to evaluate mobility ratios - and of weighted averages of fluxes. Our methodology is closer to the latter. Given the structure of the Implicit Hybrid Upwinding flux, we employ weighted averages of mobility ratios to obtain a fully implicit multidimensional IHU satisfying the monotonicity property.

\subsection{Viscous term}

In the viscous term $\bar{V}_{\ell, k+1 / 2}$, the mobility ratio at half interface $\Gamma_{k+1 / 2}$ is approximated based on the sign of the discrete total velocities in the interaction region. In this section, we assume that these total velocities have been computed and are available. Their fully implicit discretization will be reviewed in Section 4.3. We write the viscous flux at half interface $\Gamma_{k+1 / 2}$ as

$$
\bar{V}_{\ell, k+1 / 2}:=\bar{\chi}_{\ell, k+1 / 2} \bar{u}_{T, k+1 / 2} .
$$

The interfacial quantity $\bar{\chi}_{\ell, k+1 / 2}$ approximates the mobility ratio of the viscous term at half interface $\Gamma_{k+1 / 2}$ (see Fig. 2) using a weighted average of the mobility ratio evaluated in the control volume and at the previous interface to reflect the local flow pattern in the interaction region, that is,

$$
\bar{\chi}_{\ell, k+1 / 2}:= \begin{cases}\left(1-\bar{\omega}_{k+1 / 2}^{V}\right) \chi_{\ell, k}+\bar{\omega}_{k+1 / 2}^{V} \bar{\chi}_{\ell, k-1 / 2} & \text { if } \bar{u}_{T, k+1 / 2}>0 \\ \left(1-\bar{\omega}_{k+1 / 2}^{V}\right) \chi_{\ell, k+1}+\bar{\omega}_{k+1 / 2}^{V} \bar{\chi}_{\ell, k+3 / 2} & \text { otherwise, }\end{cases}
$$

where $\chi_{\ell, k}$ denotes the mobility ratio evaluated at vertex $k$ :

$$
\chi_{\ell, k}:=\frac{\lambda_{\ell, k}}{\lambda_{T, k}}=\frac{\lambda_{\ell}\left(S_{\ell, k}\right)}{\lambda_{T}\left(S_{\ell, k}\right)}
$$

The weighting coefficient $\bar{\omega}_{k+1 / 2}^{V}$ is a function of the primary variables - pressure and saturation - in the interaction region and is constructed as the ratio of the total velocities at two consecutive interfaces:

$$
\bar{\omega}_{k+1 / 2}^{V}:= \begin{cases}\varphi\left(\max \left(0, \frac{\bar{u}_{T, k-1 / 2}}{\bar{u}_{T, k+1 / 2}}\right)\right) & \text { if } \bar{u}_{T, k+1 / 2}>0 \\ \varphi\left(\max \left(0, \frac{\bar{u}_{T, k+3 / 2}}{\bar{u}_{T, k+1 / 2}}\right)\right) & \text { if } \bar{u}_{T, k+1 / 2}<0 \\ 0 & \text { otherwise, }\end{cases}
$$

where $\varphi: \mathbb{R}^{+} \mapsto[0,1]$ is a limiter function used to guarantee that $\bar{\omega}_{k+1 / 2}^{V} \in[0,1]$ and enforce monotonicity with respect to saturation. With this definition, it follows that the multidimensional viscous term (22) reduces to the viscous term in the original one-dimensional IHU scheme whenever $\varphi \equiv 0$. An illustration of the viscous upwinding is given in Fig. 2 where $\bar{u}_{T, 1+1 / 2}>0, \bar{u}_{T, 2+1 / 2}>0, \bar{u}_{T, 3+1 / 2}<0$, and $\bar{u}_{T, 4+1 / 2}$.

Different limiters have been proposed in previous work, such as the Tight Multi-D Upstream (TMU) limiter (Schneider and Raw, 1986, Hurtado et al., 2007) defined by $\varphi^{T M U}(r)=\min (1, r)$ and the Smooth Multi-D Upstream (SMU) limiter (Hurtado et al., 2007, Kozdon et al., 2009) computed with $\varphi^{S M U}(r)=$ $r /(1+r)$. For a constant (uniform) flow field, the SMU limiter corresponds to the upwind scheme of Koren (1991) and the TMU limiter corresponds to the narrow scheme of Roe and Sidilkover (1992). In the absence of buoyancy, SMU aligns the numerical diffusion tensor with the flow direction and TMU minimizes the transverse numerical diffusion (Kozdon et al., 2009). In numerical tests not included here for brevity we observed that SMU does not sufficiently reduce transverse diffusion when combined with MultiD-IHU. But, we also noted that TMU introduces a discontinuity in the derivatives of the weight $\bar{\omega}_{k+1 / 2}^{V}$ that undermines the nonlinear convergence behavior of the MultiD-IHU scheme. Therefore, we 


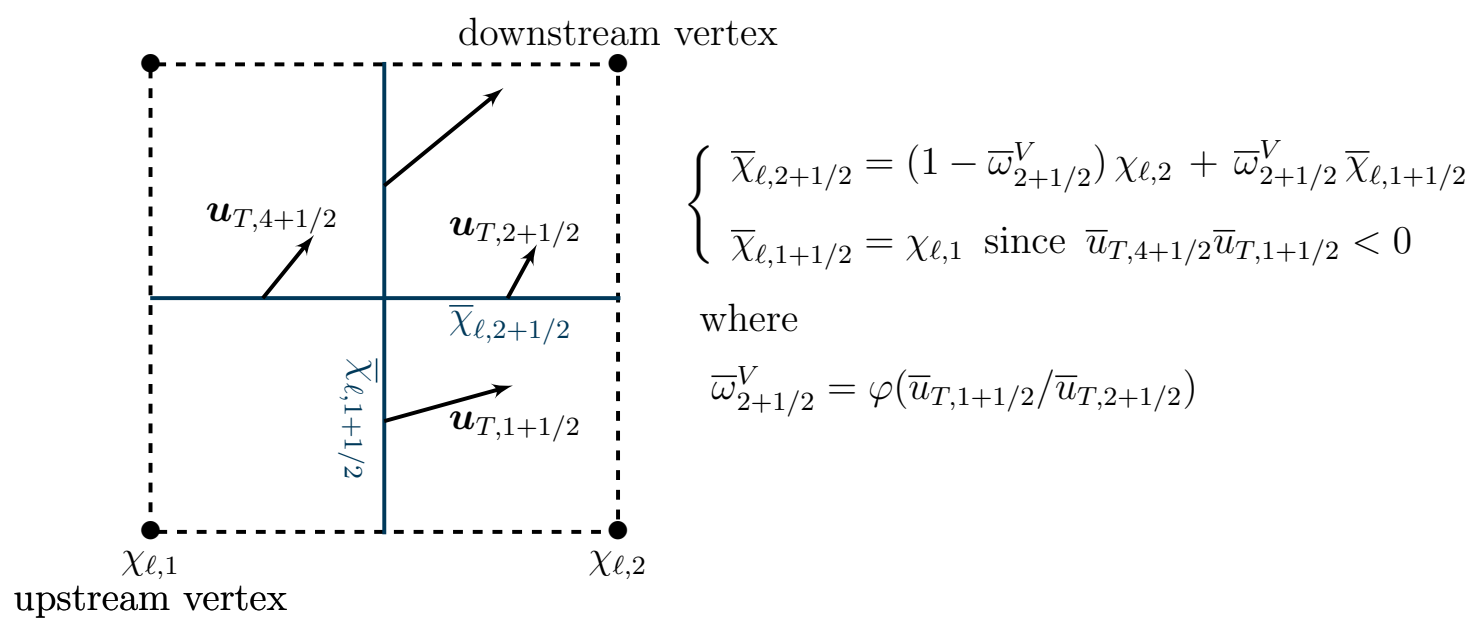

Figure 2: Viscous upwinding for half interfaces $\Gamma_{1+1 / 2}$ and $\Gamma_{2+1 / 2}$ in an interaction region. The arrows show the orientation of the total velocity at the half interfaces. The interfacial mobility, $\bar{\chi}_{\ell, k+1 / 2}$, and the vertex-based mobility ratio, $\chi_{\ell, k}$, are defined in (23) and (24), respectively.

propose a limiter that reduces transverse diffusion almost as well as TMU while preserving a robust nonlinear behavior. This variant of the SMU limiter is defined as

$$
\varphi^{S M U_{4}}(r):=\frac{r^{4}+r^{3}+r^{2}+r}{r^{4}+r^{3}+r^{2}+r+1} .
$$

This limiter, referred to as fourth-order SMU (SMU4), is shown in Fig. 3. In the MultiD-IHU scheme, we use the SMU4 limiter, that is, we set $\varphi \equiv \varphi^{S M U 4}$. We note that the SMU4 limiter satisfies the symmetry property

$$
\varphi\left(\frac{1}{r}\right)=\frac{\varphi(r)}{r} .
$$

This property, also satisfied by the SMU and TMU limiters, ensures that backward-facing and forwardfacing fluxes are treated the same way. It is also used to prove the monotonicity of the buoyancy term introduced in Section 4.2 .

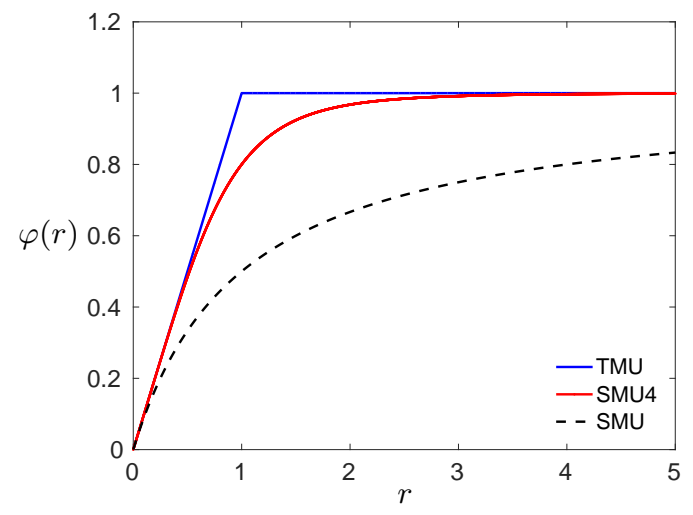

Figure 3: Multidimensional limiters discussed in this work, including the Tight Multi-D Upstream (TMU) limiter of Schneider and Raw (1986), the Smooth Multi-D Upstream (SMU) limiter of Hurtado et al. (2007), and the fourth-order SMU limiter of (26).

This methodology leads to a viscous flux that is a differentiable function of the saturations in the interaction region for a fixed total velocity field. We will show with numerical examples in Section 7 that the scheme remains well-behaved when the total velocity is a space- and time-dependent function 


\section{Hamon and Mallison}

of the primary variables. Equations 23 and 25 introduce a local coupling of the viscous terms in the interaction region that can be written in a compact matrix form as

$$
A \bar{\chi}_{\ell}=B \chi_{\ell}
$$

where $\boldsymbol{A}=\left\{a_{i j}\right\} \in \mathbb{R}^{4 \times 4}$ has unit diagonal elements $\left(a_{k k}=1\right)$ and one non-zero off-diagonal term per row defined as

$$
a_{k(k-1)}:=\left\{\begin{array}{ll}
-\bar{\omega}_{k+1 / 2}^{V} & \text { if } \bar{u}_{T, k+1 / 2} \geq 0 \\
0 & \text { otherwise. }
\end{array} \quad a_{k(k+1)}:= \begin{cases}0 & \text { if } \bar{u}_{T, k+1 / 2} \geq 0 \\
-\bar{\omega}_{k+1 / 2}^{V} & \text { otherwise }\end{cases}\right.
$$

and $\boldsymbol{B}=\left\{b_{i j}\right\} \in \mathbb{R}^{4 \times 4}$ is non-negative, such that

$$
b_{k k}:=\left\{\begin{array}{ll}
1-\bar{\omega}_{k+1 / 2}^{V} & \text { if } \bar{u}_{T, k+1 / 2} \geq 0 \\
0 & \text { otherwise. }
\end{array} \quad b_{k(k+1)}:= \begin{cases}0 & \text { if } \bar{u}_{T, k+1 / 2} \geq 0 \\
1-\bar{\omega}_{k+1 / 2}^{V} & \text { otherwise }\end{cases}\right.
$$

In Appendix A, we use the definitions given in 26 to 30 to prove that $\boldsymbol{A}$ is a non-singular M-matrix. We also show that the matrix $\boldsymbol{C}:=\boldsymbol{A}^{-1} \boldsymbol{B}$ is non-negative and satisfies

$$
\sum_{j} c_{i j}=1 \quad i \in\{1, \ldots, 4\}
$$

The interfacial mobility ratios are then determined by solving the following local $(4 \times 4)$ linear system

$$
\bar{\chi}_{\ell}=\boldsymbol{A}^{-1} \boldsymbol{B} \chi_{\ell}
$$

With this approach, each interfacial mobility ratio can depend on more than two saturations in the interaction region. This leads to an improved accuracy when the flow is not perpendicular to the interface while preserving the monotonicity of the viscous flux in the sense of (18), as proven in Appendix B. We mention here that the matrices $\boldsymbol{A}$ and $\boldsymbol{B}$ are also used to obtain the derivatives of the mobility ratios with respect to pressure and saturation as shown below,

$$
\frac{\partial \overline{\boldsymbol{\chi}}_{\ell}}{\partial \tau_{k}}=\boldsymbol{A}^{-1}\left(-\frac{\partial \boldsymbol{A}}{\partial \tau_{k}} \overline{\boldsymbol{\chi}}_{\ell}+\frac{\partial \boldsymbol{B}}{\partial \tau_{k}} \boldsymbol{\chi}_{\ell}+\boldsymbol{B} \frac{\partial \boldsymbol{\chi}_{\ell}}{\partial \tau_{k}}\right)
$$

where $\tau_{k}$ represents the pressure at vertex $k, p_{k}$, or the wetting-phase saturation at vertex $k, S_{k}$. The matrices $\partial \boldsymbol{A} / \partial \tau_{k}$ and $\partial \boldsymbol{B} / \partial \tau_{k}$ contain the derivatives of the coefficients of $\boldsymbol{A}$ and $\boldsymbol{B}$, respectively. Finally, we highlight that at each half interface, we still recover the discrete total velocity when we sum of the viscous terms over the two phases. That is, for interface $\Gamma_{k+1 / 2}$, we have

$$
\sum_{\ell} \bar{V}_{\ell, k+1 / 2}=\left(\sum_{\ell} \bar{\chi}_{\ell, k+1 / 2}\right) \bar{u}_{T, k+1 / 2}=\left(\sum_{j} c_{i j}\left(\sum_{\ell} \chi_{\ell, j}\right)\right) \bar{u}_{T, k+1 / 2}=\bar{u}_{T, k+1 / 2}
$$

where the rightmost equality is obtained using 24 and 31 .

\subsection{Buoyancy term}

In the buoyancy term $\bar{G}_{\ell, k+1 / 2}$, the discretization of the mobility ratio at half interface $\Gamma_{k+1 / 2}$ is fully based on (fixed) density differences as in the one-dimensional IHU scheme. Our approach is based on the fact that the heaviest (respectively, lightest) phase propagates downwards (respectively, upwards). We write the buoyancy term as

$$
\bar{G}_{\ell, k+1 / 2}:=\bar{T}_{k+1 / 2} \sum_{m} \bar{\psi}_{\ell, m, k+1 / 2}\left(\rho_{\ell}-\rho_{m}\right) g \overline{\Delta z}_{k+1 / 2}
$$

where $\overline{\Delta z}_{k+1 / 2}=z_{k+1}-z_{k}$, and where $\bar{T}_{k+1 / 2}$ is defined in 16 . We omitted again the superscript denoting the interaction region in the right-hand side. As in the viscous term, the discrete mobility ratio 
at half interface $\Gamma_{k+1 / 2}$ between vertices $k$ and $k+1$ is obtained with a weighted averaging procedure that accounts for the orientation of the buoyancy force with respect to the grid. This procedure is described below for the case $\left(\rho_{\ell}-\rho_{m}\right) \overline{\Delta z}_{k+1 / 2}>0$ :

$$
\bar{\psi}_{\ell, m, k+1 / 2}:= \begin{cases}\left(1-\bar{\omega}_{k+1 / 2}^{G}\right) \frac{\lambda_{\ell, k} \lambda_{m, k+1}}{\lambda_{\ell, k}+\lambda_{m, k+1}}+\bar{\omega}_{k+1 / 2}^{G} \frac{\lambda_{\ell, k} \lambda_{m, k+2}}{\lambda_{\ell, k}+\lambda_{m, k+2}} & \text { if } \overline{\Delta z}_{k+1 / 2} \overline{\Delta z}_{k+3 / 2}>0 \\ \left(1-\bar{\omega}_{k+1 / 2}^{G}\right) \frac{\lambda_{\ell, k} \lambda_{m, k+1}}{\lambda_{\ell, k}+\lambda_{m, k+1}}+\bar{\omega}_{k+1 / 2}^{G} \frac{\lambda_{\ell, k-1} \lambda_{m, k+1}}{\lambda_{\ell, k-1}+\lambda_{m, k+1}} & \text { if } \overline{\Delta z}_{k+1 / 2} \overline{\Delta z}_{k-1 / 2}>0 \\ \frac{\lambda_{\ell, k} \lambda_{m, k+1}}{\lambda_{\ell, k}+\lambda_{m, k+1}} & \text { otherwise. }\end{cases}
$$

The following cases are illustrated in Fig. 4 for a light non-wetting phase and a heavy wetting phase. First, we consider updip half interface $\Gamma_{2+1 / 2}\left(\overline{\Delta z}_{2+1 / 2}<0\right)$. Since $\Gamma_{1+1 / 2}$ is downdip and $\Gamma_{3+1 / 2}$ is updip, the non-wetting phase mobility, $\lambda_{n w}$, is evaluated at the bottom vertex 2 whereas the wetting-phase mobility $\lambda_{w}$ is evaluated at vertex 3 and at the top vertex 4 . Second, we consider updip half interface $\Gamma_{3+1 / 2}$ $\left(\overline{\Delta z}_{3+1 / 2}<0\right)$. Since $\Gamma_{4+1 / 2}$ is downdip and $\Gamma_{2+1 / 2}$ is updip, $\lambda_{n w}$ is evaluated at vertex 3 and at the bottom vertex 2 while $\lambda_{w}$ is evaluated at the top vertex 1 .

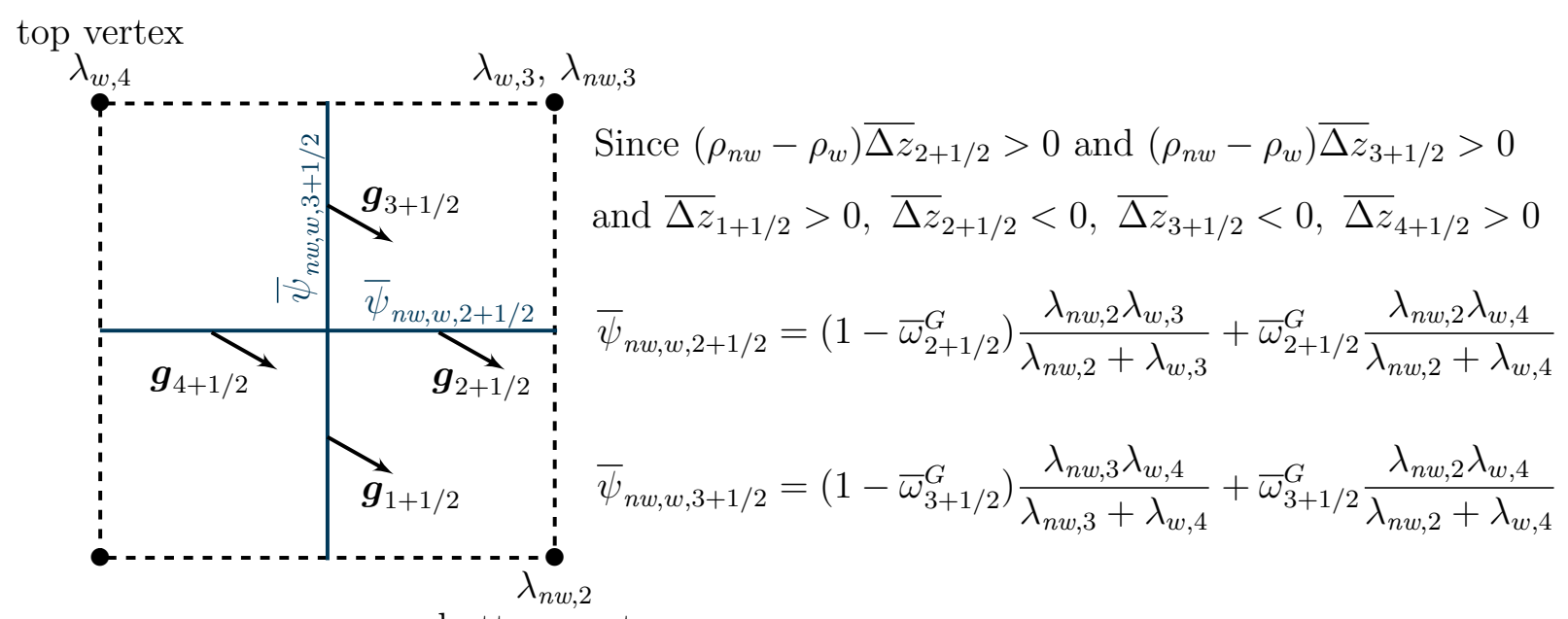

bottom vertex

Figure 4: Buoyancy upwinding for half interfaces $\Gamma_{2+1 / 2}$ and $\Gamma_{3+1 / 2}$ in an interaction region. The arrows indicate the gravity vector at each half interface. For this configuration, vertex 4 is at the top and vertex 2 is at the bottom. Therefore, we have $\overline{\Delta z}_{1+1 / 2}=z_{2}-z_{1}>0, \overline{\Delta z}_{2+1 / 2}=z_{3}-z_{2}<0, \overline{\Delta z}_{3+1 / 2}=z_{4}-z_{3}<0$, and $\overline{\Delta z}_{4+1 / 2}=z_{1}-z_{4}>0$. We consider a light non-wetting phase denoted by the subscript nw and a heavy wetting phase denoted by the subscript $w$, such that $\rho_{n w}-\rho_{w}<0$. The interfacial mobility ratio, $\bar{\psi}_{n w, w, k+1 / 2}$, is defined in (36).

The case $\left(\rho_{\ell}-\rho_{m}\right) \overline{\Delta z}_{k+1 / 2} \leq 0$ is treated analogously and is obtained by switching the phase indices $\ell$ and $m$ in 36 . Finally, the weighting coefficient $\bar{\omega}_{k+1 / 2}^{G}$ is written as

$$
\bar{\omega}_{k+1 / 2}^{G}:= \begin{cases}\varphi\left(\frac{\left.\bar{T}_{k+3 / 2} \overline{\Delta z}_{k+3 / 2}\right)}{\bar{T}_{k+1 / 2} \overline{\Delta z}_{k+1 / 2}}\right) & \text { if } \overline{\Delta z}_{k+1 / 2} \overline{\Delta z}_{k+3 / 2}>0 \\ \varphi\left(\frac{\bar{T}_{k-1 / 2} \overline{\Delta z}_{k-1 / 2}}{\bar{T}_{k+1 / 2} \overline{\Delta z}_{k+1 / 2}}\right) & \text { if } \overline{\Delta z}_{k+1 / 2} \overline{\Delta z}_{k-1 / 2}>0 \\ 0 & \text { otherwise, }\end{cases}
$$

where $\varphi$ is defined in 26 and guarantees that $\bar{\omega}_{k+1 / 2}^{G} \in[0,1]$. The multidimensional buoyancy term reduces to the one-dimensional IHU buoyancy term whenever $\varphi \equiv 0$. In the compressible case, the ratio of (37) would also contain phase densities. These densities cancel in the incompressible case considered in this work and are omitted for clarity. 


\section{Hamon and Mallison}

Unlike the viscous term, the buoyancy term does not require a local linear solve to determine the mobility ratio since $\bar{\psi}_{\ell, m, k+1 / 2}$ is decoupled from $\bar{\psi}_{\ell, m, k-1 / 2}$ and $\bar{\psi}_{\ell, m, k+3 / 2}$. By construction, $\bar{G}_{\ell, k+1 / 2}$ is a differentiable function of the saturations, and its derivatives can be computed in a straightforward manner. We show in Appendix C that since the SMU4 limiter satisfies the symmetry property (27), the buoyancy term constitutes a monotone approximation of the buoyancy flux in the sense of (18). Since the same property holds for the viscous term, 21 implies that $\bar{F}_{\ell, k+1 / 2}$ satisfies the monotonicity condition of 18 . Finally, we note that by construction, we have $\bar{\psi}_{\ell, m, k+1 / 2}=\bar{\psi}_{m, \ell, k+1 / 2}$. It follows that

$$
\bar{G}_{\ell, k+1 / 2}=-\bar{G}_{m, k+1 / 2}
$$

\subsection{Total velocity discretization}

In the previous paragraphs, the presentation has focused on the discretization of the mobilities present in the transport equation. To complete the formulation of the multidimensional IHU scheme, we now describe the computation of the total velocity. Before this, we note that (15) does not provide a practical way to compute $\bar{u}_{T, k+1 / 2}$, as this quantity is needed to evaluate the phase fluxes present in the right-hand side. Instead, equation (15) imposes a consistency requirement on the transport scheme. This consistency is satisfied by the methodology presented in Sections 4.1, 4.2, as shown by (34) and (38).

Considering a half interface $\Gamma_{k+1 / 2}$ between vertices $k$ and $k+1$, we compute the total velocity, $\bar{u}_{T, k+1 / 2}$, as

$$
\bar{u}_{T, k+1 / 2}\left(\overline{\Delta p}_{k+1 / 2}, S_{k}, S_{k+1}\right):=\bar{T}_{k+1 / 2} \bar{\lambda}_{T, k+1 / 2}^{W A} \overline{\Delta p}_{k+1 / 2}+\bar{T}_{k+1 / 2} \sum_{\ell} \bar{\lambda}_{\ell, k+1 / 2}^{W A} \rho_{\ell} g \overline{\Delta z}_{k+1 / 2},
$$

where $\bar{\lambda}_{\ell, k+1 / 2}^{W A}$ denotes the discrete mobility of phase $\ell$ at half interface $\Gamma_{k+1 / 2}$. To compute this quantity, we apply a differentiable discretization derived in Hamon et al. (2016) that aims at attenuating the flipflopping in the total velocity to improve the nonlinear convergence of the scheme. This methodology is based on an averaging procedure in which the mobility at half interface $\Gamma_{k+1 / 2}$ in $(39)$ is defined as

$$
\bar{\lambda}_{\ell, k+1 / 2}^{W A}:=\bar{\beta}_{k+1 / 2}\left(\overline{\Delta p}_{k+1 / 2}\right) \lambda_{\ell}\left(S_{k}\right)+\left(1-\bar{\beta}_{k+1 / 2}\left(\overline{\Delta p}_{k+1 / 2}\right)\right) \lambda_{\ell}\left(S_{k+1}\right) .
$$

The weighting coefficient $\bar{\beta}_{k+1 / 2} \in[0,1]$ is designed such that the resulting phase mobilities are differentiable and increasing functions of the pressure difference that adapt to the balance between viscous and buoyancy forces at each interface in the computational domain. It reads

$$
\bar{\beta}_{k+1 / 2}\left(\overline{\Delta p}_{k+1 / 2}\right):=\frac{1}{2}+\frac{1}{\pi} \arctan \left(\bar{\gamma}_{k+1 / 2} \overline{\Delta \Phi}_{k+1 / 2}\right) .
$$

The coefficient $\bar{\gamma}_{k+1 / 2} \in \mathbb{R}^{+}$relates the magnitude of viscous forces to the magnitude of buoyancy forces and is given in Hamon et al. (2016). In $41, \overline{\Delta \Phi}_{k+1 / 2}$ denotes the average potential gradient at the interface, defined by:

$$
\overline{\Delta \Phi}_{k+1 / 2}:=\frac{1}{n_{p}} \sum_{\ell} \overline{\Delta \Phi}_{\ell, k+1 / 2}=\overline{\Delta p}_{k+1 / 2}+\frac{1}{n_{p}} \sum_{\ell} \rho_{\ell} g \overline{\Delta z}_{k+1 / 2} .
$$

We emphasize the fact that the only difference between the standard PPU discretization of the total velocity used, for instance, in Lee and Efendiev (2018), and our methodology is the discretization of the mobilities described in (40) to (42). In previous work (Hamon et al., 2016), we found that the nonlinear behavior improves significantly when the mobilities are discretized with (40) to 42 and we proved that the resulting discrete pressure equation remains well behaved with this approach.

\section{Mathematical properties of the scheme}

Here, we analyze the fully implicit scheme for coupled flow and transport constructed above. We consider the system of governing equations given in Section 3, with a discrete transport equation (13) for one of the phases (here, the wetting phase $\ell=w$ ) and a discrete pressure equation (15). The total velocity is discretized as explained in Section 4.3. Next, we use the monotonicity condition satisfied by the multidimensional IHU numerical flux to show that the saturation solution remains between 0 and 1. 
Proposition 1. (Saturation estimate) Consider the pressure-saturation solution to the fully implicit scheme defined in (13)-(15) with $q_{\ell}=0$. Provided that the initial saturation is between physical bounds, we have the following saturation estimate, valid for all interaction regions in the domain and all time levels $n \in \mathbb{N}^{+}$:

$$
0 \leq\left(S_{k}^{(m)}\right)^{n} \leq 1
$$

where $k$ denotes the local index of the vertices in interaction region $(m)$.

Proof. The proof is done by induction on the time level $n$ and relies on the monotonicity of the flux in the sense of (18). We adapt a proof from Brenner et al. (2013) also used in Hamon et al. (2018).

We assume that (43) holds for all interaction regions for time levels $n \leq n_{0}$. To prove that the solution of the scheme also satisfies this inequality at time level $n_{0}+1$, we first consider the blue control volume of Fig. 1. Unless stated otherwise, all the quantities in the proof are evaluated fully implicitly at time level $n_{0}+1$. In interaction region (2), the net wetting-phase flux at the two half interfaces $\Gamma_{3+1 / 2}^{(2)}$ and $\Gamma_{4+1 / 2}^{(2)}$ is a function of the total velocity field in interaction region (2), denoted by $\bar{u}_{T, k+1 / 2}^{(2)}, k \in\{1, \ldots, 4\}$, and the saturations used in the evaluation of the viscous and buoyancy mobility ratios of (23) and (36), denoted by $S_{k}^{(2)}$. Isolating the saturation at the center of the blue control volume - or, equivalently, the saturation at vertex 4 in interaction region $(2)$ - we write the net flux at $\Gamma_{3+1 / 2}^{(2)}$ and $\Gamma_{4+1 / 2}^{(2)}$, denoted by $\bar{F}^{(2)}$, as

$$
\bar{F}_{w}^{(2)}:=\left(\bar{F}_{w, 4+1 / 2}^{(2)}-\bar{F}_{w, 3+1 / 2}^{(2)}\right)\left(\bar{u}_{T, l+1 / 2, l \in\{1, \ldots, 4\}}^{(2)}, S_{4}^{(2)}, S_{l \neq 4}^{(2)}\right) .
$$

Now, we take the maximum between $S_{l}^{(2)}$ and 1 at vertices $l \neq 4$ in 44 . Using the monotonicity of the net flux given in 18 , we obtain

$$
\left(\bar{F}_{w, 4+1 / 2}^{(2)}-\bar{F}_{w, 3+1 / 2}^{(2)}\right)\left(\bar{u}_{T, l+1 / 2, l \in\{1, \ldots, 4\}}^{(2)}, S_{4}^{(2)}, \max \left(S_{l}^{(2)}, 1\right)_{l \neq 4}\right) \leq \bar{F}_{w}^{(2)} .
$$

We note that an analogous result holds for interaction regions (1), (3), and (4). Now, we consider the accumulation term in the blue control volume of Fig. 11 still viewed from interaction region (2). Using the induction hypothesis, one can write

$$
V \phi \frac{S_{4}^{(2)}-1}{\Delta t^{n}} \leq V \phi \frac{S_{4}^{(2)}-\left(S_{4}^{(2)}\right)^{n_{0}}}{\Delta t^{n}} .
$$

Since we are considering the solution of the system at time $n_{0}+1$, it satisfies the discrete transport equation (13). Using this remark, we combine (45) written in the four interaction regions and 46] to obtain

$$
V \phi \frac{S_{4}^{(2)}-1}{\Delta t^{n}}+\sum_{k}\left(\bar{F}_{w, k+1 / 2}^{(k-2)}-\bar{F}_{w, k-1 / 2}^{(k-2)}\right)\left(\bar{u}_{T, l+1 / 2, l \in\{1, \ldots, 4\}}^{(k-2)}, S_{k}^{(k-2)}, \max \left(S_{l}^{(k-2)}, 1\right)_{l \neq k}\right) \leq 0 .
$$

Before proceeding to the next step, we remind the reader that in the notation detailed in Fig. 1. $S_{3}^{(1)}, S_{4}^{(2)}$, $S_{1}^{(3)}$, and $S_{4}^{(2)}$ denote the same degree of freedom, $S_{k}^{(k-2)}$, which refers to the wetting-phase saturation at the center of the control volume in blue.

Next, we consider the discrete pressure equation in the blue control volume of Fig. 1 First, assume that for the total velocity field satisfying the discrete pressure equation 15 , we evaluate the viscous mobility ratios of 233 and the buoyancy mobility ratio of (36) with unit wetting-phase saturations in the interaction region, i.e., $S_{l}^{(2)}=1$ for $l \in\{1, \ldots, 4\}$. In this configuration, we have $\bar{\chi}_{w, l+1 / 2}^{(2)}=1$ and $\bar{\psi}_{w, l+1 / 2}^{(2)}=0$ for $l \in\{1, \ldots, 4\}$. This implies that

$$
\begin{aligned}
\left(\bar{F}_{w, 4+1 / 2}^{(2)}-\bar{F}_{w, 3+1 / 2}^{(2)}\right)\left(\bar{u}_{T, l+1 / 2, l \in\{1, \ldots, 4\}}^{(2)}, 1,(1)_{l \neq 4}\right) & =\bar{\chi}_{w, 4+1 / 2}^{(2)} \bar{u}_{T, 4+1 / 2}^{(2)}-\bar{\chi}_{w, 3+1 / 2}^{(2)} \bar{u}_{T, 3+1 / 2}^{(2)} \\
& +\bar{\psi}_{w, n w, 4+1 / 2}^{(2)}-\bar{\psi}_{w, n w, 3+1 / 2}^{(2)} \\
& =\bar{u}_{T, 4+1 / 2}^{(2)}-\bar{u}_{T, 3+1 / 2}^{(2)} .
\end{aligned}
$$




\section{Hamon and Mallison}

Next, we resort to the procedure employed to obtain (45). That is, in (48), we take the maximum between $S_{l}^{(2)}$ and 1 at vertices $l \neq 4$ and use the monotonicity of the numerical flux in the sense of 18 to obtain

$$
\left(\bar{F}_{w, 4+1 / 2}^{(2)}-\bar{F}_{w, 3+1 / 2}^{(2)}\right)\left(\bar{u}_{T, l+1 / 2, l \in\{1, \ldots, 4\}}^{(2)}, 1, \max \left(S_{l}^{(2)}, 1\right)_{l \neq 4}\right) \leq \bar{u}_{T, 4+1 / 2}^{(2)}-\bar{u}_{T, 3+1 / 2}^{(2)}
$$

An analogous inequality holds for interaction regions (1), (3), and (4), respectively. Since the total velocity field satisfies the discrete pressure equation $(15)$, we obtain

$$
\sum_{k}\left(\bar{F}_{w, k+1 / 2}^{(k-2)}-\bar{F}_{w, k-1 / 2}^{(k-2)}\right)\left(\bar{u}_{T, l+1 / 2, l \in\{1, \ldots, 4\}}^{(k-2)}, 1, \max \left(S_{l}^{(k-2)}, 1\right)_{l \neq k}\right) \leq 0
$$

We now consider 477 and 50 . Since $S_{2}^{(k-2)}$ for different $k \in\{1, \ldots, 4\}$ refers to the same degree of freedom, that is, the saturation at the center of the blue control volume in Fig. 1) we see that (47) and (50) only differ by one argument in the flux term - in the second slot - and in the accumulation term in the saturation at the most recent time level. This argument is equal to $S_{4}^{(2)}$ in 47 ) and to 1 in (50). In the next equation, we combine (47) and (50) by taking the maximum of $S_{4}^{(2)}$ and 1 which leads to

$$
\begin{aligned}
V & \phi \frac{\max \left(S_{4}^{(2)}, 1\right)-1}{\Delta t^{n}} \\
& +\sum_{k}\left(\bar{F}_{w, k}^{(k-2)}-\bar{F}_{w, k-1}^{(k-2)}\right)\left(\bar{u}_{T, l+1 / 2, l \in\{1, \ldots, 4\}}^{(k-2)}, \max \left(S_{k}^{(k-2)}, 1\right), \max \left(S_{l}^{(k-2)}, 1\right)_{l \neq k}\right) \leq 0 .
\end{aligned}
$$

We see that we recover 47 whenever $S_{4}^{(2)}>1$, and we recover 50 whenever $S_{4}^{(2)} \leq 1$. To obtain (51), we have considered the blue control volume in Fig. 1. but this inequality is valid for all the control volumes in the domain. Therefore, we can sum this inequality over all control volumes in the domain. In the result inequality, we slightly abuse the notation and now denote by $S_{i}$ the saturation at the center of control volume $i$. Since the scheme is mass conservative and the fluxes are evaluated with the same arguments, the flux terms cancel and we are left with

$$
\sum_{i} V_{i} \phi_{i} \frac{\max \left(S_{i}, 1\right)-1}{\Delta t^{n}} \leq 0 .
$$

which gives $S_{i} \leq 1$ for all control volumes. Therefore, we have proven the rightmost inequality in (43). The proof for $S_{i} \geq 0$ is analogous.

\section{Newton's method with damping}

The systems of nonlinear algebraic equations of $132-(15)$ are solved with Newton's method with damping (Deuflhard, 2011). Successive linearizations and updates are performed until convergence is reached:

$$
\begin{array}{ll}
\text { solve } & \boldsymbol{J} \delta \boldsymbol{U}^{\nu+1}=-\boldsymbol{R}\left(\boldsymbol{U}^{n+1, \nu}\right) \text { for } \delta \boldsymbol{U}^{\nu+1}, \\
\text { then } & \boldsymbol{U}^{n+1, \nu+1} \leftarrow \boldsymbol{U}^{n+1, \nu}+\boldsymbol{\tau}^{\nu+1} \delta \boldsymbol{U}^{\nu+1},
\end{array}
$$

where $n$ denotes the time level, $\nu$ denotes the nonlinear iteration number, $\boldsymbol{\tau}^{\nu+1} \in \mathbb{R}^{N n_{p} \times N n_{p}}$ is a diagonal matrix of damping parameters, and $\boldsymbol{J} \in \mathbb{R}^{N n_{p} \times N n_{p}}$ denotes the Jacobian matrix of $\boldsymbol{R}$ with respect to the primary variables. In [54), the diagonal term of a pressure row of $\left.\left.\boldsymbol{\tau}^{\nu+1} \in\right] 0,1\right]$ is equal to one. The diagonal term of a saturation row is equal to one if the local absolute saturation update, $\left|\delta S^{\nu+1}\right|$, is smaller than $(\delta S)_{\max }:=0.2$. Otherwise, this diagonal term is set to $(\delta S)_{\max } /\left|\delta S^{\nu+1}\right|$. We consider the control volume in blue in Fig. 1 in which the saturation of phase $\ell$ viewed from interaction region (2) is denoted by $S_{\ell, 4}^{(2)}$. The convergence criterion in this control volume is satisfied when the value of the normalized residual drops below the tolerance for the two phases:

$$
\frac{V \phi\left(S_{\ell, 4}^{(2)}-\left(S_{\ell, 4}^{(2)}\right)^{n}\right)+\Delta t^{n}\left(\sum_{k}\left(\bar{F}_{\ell, k+1 / 2}^{(k-2)}-\bar{F}_{\ell, k-1 / 2}^{(k-2)}\right)-V q_{\ell}\right)}{V \phi} \leq 10^{-8}, \quad \ell \in\{n w, w\} .
$$




\section{Fully Implicit Multidimensional Hybrid Upwind Scheme}

Convergence is reached when (55) is satisfied in all control volumes. If the solver fails to converge after 50 iterations, Newton's method is restarted from the previous time step, with a time step size reduced by half. If necessary, this time step chopping is repeated. After a converged solution is obtained, the time step size is reset to its original size.

\section{Numerical examples}

In this section, we compare the accuracy and performance of four fully implicit schemes. The first scheme, referred to as 1D-PPU, is based on the standard two-point phase-per-phase upwinding commonly used in industrial simulators. 1D-IHU is the scheme proposed in Hamon et al. (2016). It uses a twopoint hybrid upwinding combined with the procedure based on a weighted averaging of the mobilities in the total velocity. MultiD-PPU is the multidimensional scheme described in Kozdon et al. (2011a) constructed with the SMU limiter. Finally, MultiD-IHU is the multidimensional scheme based on hybrid upwinding described in Section 3 . It relies on the SMU4 limiter for the viscous and buoyancy fluxes.

\subsection{Three-well problem with buoyancy}

We first assess the reduction of the numerical bias caused by the orientation of the grid with a threewell problem with buoyancy derived from Kozdon et al. (2011a); Keilegavlen et al. (2012). We consider a $x-y$ domain of dimensions $[-0.5 \mathrm{ft}, 0.5 \mathrm{ft}] \times[-0.5 \mathrm{ft}, 0.5 \mathrm{ft}]$, discretized with a $51 \times 51$ uniform Cartesian grid so that $\Delta x=\Delta y=1 / 51 \mathrm{ft}$. The scalar absolute permeability is equal to

$$
k(x, y)= \begin{cases}50 \mathrm{mD} & \text { if } r=\sqrt{x^{2}+y^{2}}<r_{0} \\ 5 \times 10^{-5} \mathrm{mD} & \text { otherwise }\end{cases}
$$

where $r_{0}=(1-\Delta x) / 2$. The well locations are computed using a secondary coordinate system defined as $\left(x^{\prime}, y^{\prime}\right)=(x \cos \theta+y \sin \theta,-x \sin \theta+y \cos \theta)$. The injector is always in the center of the domain at $\left(x^{\prime}, y^{\prime}\right)=(0,0)$ and is operated at a fixed rate. The two producers are placed at $\left(x^{\prime}, y^{\prime}\right)=$ $( \pm 0.3 \sin (\pi / 6),-0.3 \cos (\pi / 6))$ and are both operated using a bottom-hole-pressure control. The symmetry of the flow pattern is preserved by orienting the buoyancy force in the direction that is perpendicular to the straight line connecting the two producers, going updip from the injector to the producers. The phase properties are such that the non-wetting phase is twice as light as the wetting phase, with $\rho_{n w}=32 \mathrm{lb}_{m} . \mathrm{ft}^{-3}$ and $\rho_{w}=64 \mathrm{lb}_{m} . \mathrm{ft}^{-3}$. The constant phase viscosities are chosen to be $\mu_{n w}=100 \mathrm{cP}$ and $\mu_{w}=1 \mathrm{cP}$. Finally, we use Corey-type relative permeabilities such that $k_{r n w}(S)=(1-S)^{4}$ and $k_{r w}(S)=S^{2}$. The gravity number is such that

$$
N_{G}:=\frac{k\left|g_{w}-g_{n w}\right|}{\mu_{w}\left|u_{T}\right|}=1.3 .
$$

The saturation maps for different angles $\theta$ at $T=0.092 \mathrm{PVI}$ are in Fig. 5 . The constant time step size is $\Delta t \approx 0.0013 \mathrm{PVI}$ and corresponds to a CFL number of approximately 3.4. The saturation maps show that the 1D-PPU and 1D-IHU schemes are very sensitive to the orientation of the grid as they prefer flow along the grid directions. The proposed MultiD-IHU scheme does not exhibit this severe numerical bias and significantly reduces the grid orientation effect. We note that the MultiD-IHU saturation maps are in agreement with those generated with MultiD-PPU. These findings are confirmed by the water cuts at the two producers in Fig. 6. They show that the truly multidimensional schemes yield a consistent value of the breakthrough time at the producers when the grid is rotated, and better preserve the symmetry of the problem. This is not the case for 1D-PPU and 1D-IHU, which both predict very different water cuts for the two producers.

The nonlinear behavior for different angles is summarized in Table1. We run the simulations with a small time step size leading to a CFL number of 3.4, and with a larger time step size corresponding to a CFL number of 59.7. Given that we have set the maximum number of Newton iterations to a large value - i.e., 50 -, none of the schemes require time step cuts. As in Kozdon et al. (2011a), we observe that the multidimensional schemes require fewer Newton iterations than the one-dimensional schemes, even though the cost per iteration of the multidimensional schemes is significantly larger. With MultiD-IHU, the reduction in the number of iterations summed over the five cases compared to $1 \mathrm{D}-\mathrm{PPU}$ is $12.6 \%$ for a CFL number of 3.4, and reaches $12.3 \%$ for a larger CFL number of 59.7. We finally observe that MultiD-IHU consistently converges faster than MultiD-PPU in both time stepping configurations. 


\section{Hamon and Mallison}

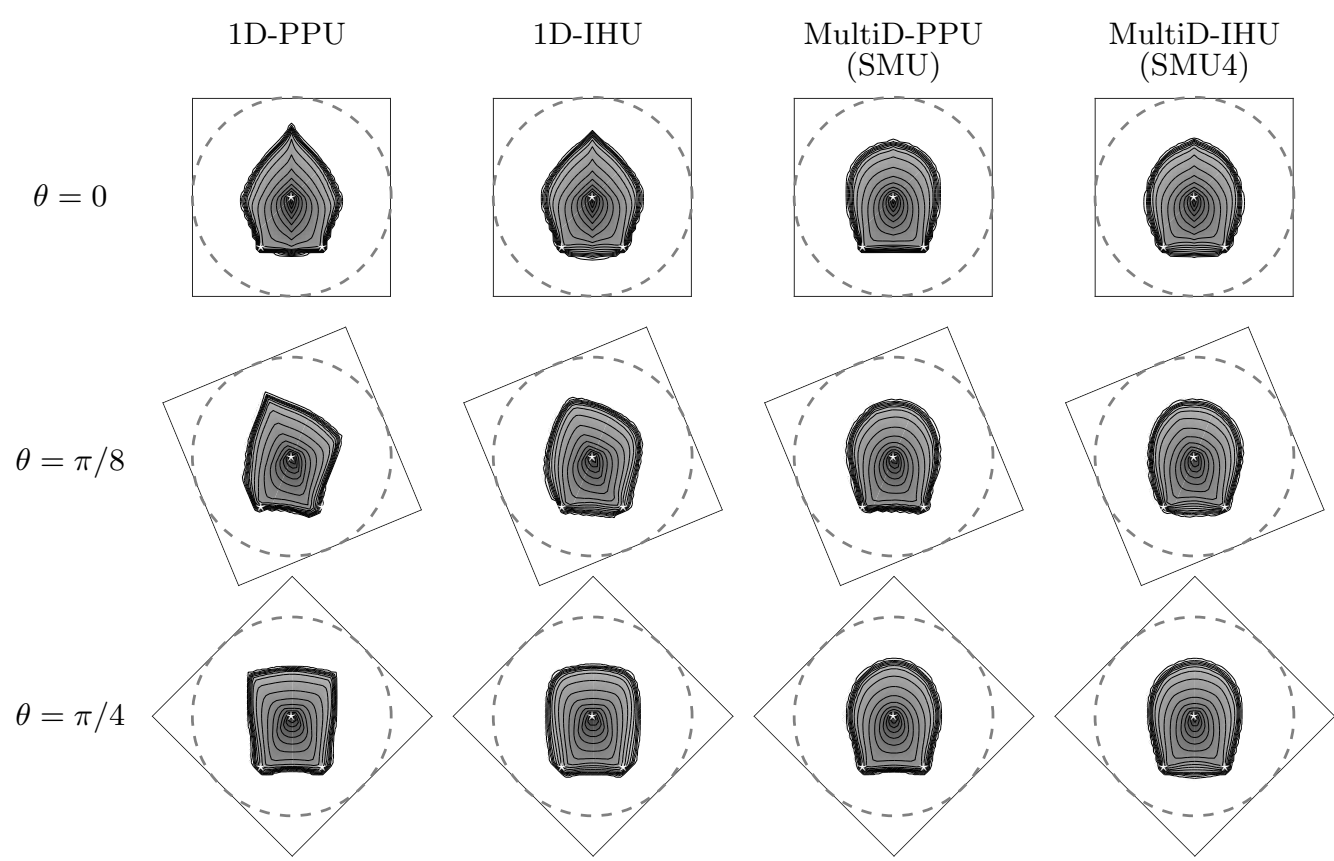

Figure 5: Saturation maps for different angles $\theta$ at $T=0.092$ PVI in the three-well problem with buoyancy. The CFL number of these simulations is 3.4. Outside the dashed circle, the control volumes have an absolute permeability set to $5 \times 10^{-5} \mathrm{mD}$. The white stars show the location of the wells.

\begin{tabular}{ccccccccc}
\hline $\begin{array}{c}\text { Angle } \\
\theta\end{array}$ & \multicolumn{3}{c}{ Small CFL } & \multicolumn{4}{c}{ Large CFL } \\
\hline 0 & 267 & 253 & 241 & 231 & 44 & 44 & 40 & 40 \\
$\pi / 12$ & 270 & 258 & 244 & 234 & 47 & 48 & 43 & 42 \\
$\pi / 8$ & 270 & 259 & 244 & 236 & 48 & 47 & 42 & 40 \\
$\pi / 6$ & 270 & 258 & 248 & 239 & 48 & 47 & 43 & 41 \\
$\pi / 4$ & 267 & 245 & 243 & 234 & 48 & 46 & 43 & 43 \\
\hline
\end{tabular}

Table 1: Total number of Newton iterations for different angles at $T=0.092$ PVI in the three-well problem with buoyancy. There was no time step cut for any of the schemes considered here.

\subsection{Two-phase flow in a heterogeneous medium}

Here, we evaluate the reduction of the grid orientation effect achieved with MultiD-IHU on a heterogeneous porous medium. The two-dimensional $x-y$ domain of dimensions $[-75 \mathrm{ft}, 75 \mathrm{ft}] \times[-75 \mathrm{ft}, 75 \mathrm{ft}]$ is discretized with $101 \times 101$ uniform Cartesian control volumes. Outside a disc of radius $r_{0}=(150-$ $\Delta x) / 2 \mathrm{ft}$ centered in $\left(x_{0}, y_{0}\right)=\left(x_{0}^{\prime}, y_{0}^{\prime}\right)=(0,0)$, we set a constant absolute permeability of $10^{-10} \mathrm{mD}$. Inside the disc, the permeability field is given by

$$
k\left(x^{\prime}, y^{\prime}\right)=200\left(1+\left(\cos \left(3 x^{\prime} \pi\right) \cos \left(3 y^{\prime} \pi\right) \cos (3 \bar{x} \pi) \cos (3 \bar{y} \pi)\right) / 2\right)^{3},
$$

where

$$
\bar{x}=x^{\prime} \cos \left(\frac{\pi}{4}\right)+y^{\prime} \sin \left(\frac{\pi}{4}\right) \quad \text { and } \quad \bar{y}=-x^{\prime} \sin \left(\frac{\pi}{4}\right)+y^{\prime} \cos \left(\frac{\pi}{4}\right) .
$$

The permeability map obtained from (58) can be seen in Fig. 7(a). It exhibits eight preferential paths originating from the center of the domain. This map is rotated to test the sensitivity of the schemes to the orientation of the grid using the same technique as in Section 7.1. The height of each control volume in the domain is a function of the radius $r=\sqrt{x^{\prime 2}+y^{\prime 2}}$, computed with

$$
h\left(x^{\prime}, y^{\prime}\right)=h_{\text {ref }}+h_{\text {bump }}\left(x^{\prime}, y^{\prime}\right) \quad \text { where } \quad h_{\text {bump }}\left(x^{\prime}, y^{\prime}\right)=20 \sin \left(\left(1+\min \left(1, \frac{r}{r_{0}}\right)\right) \frac{\pi}{2}\right),
$$




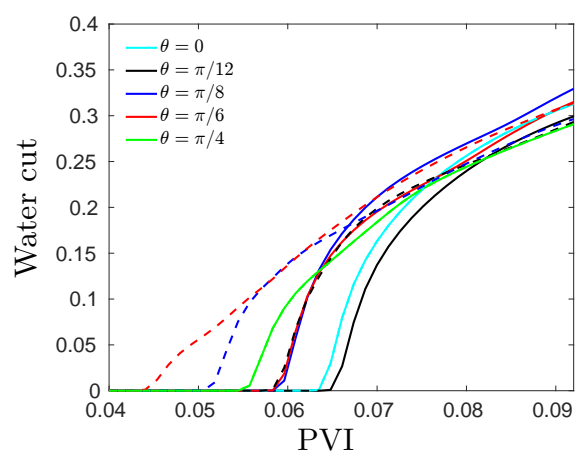

(a) 1D-PPU

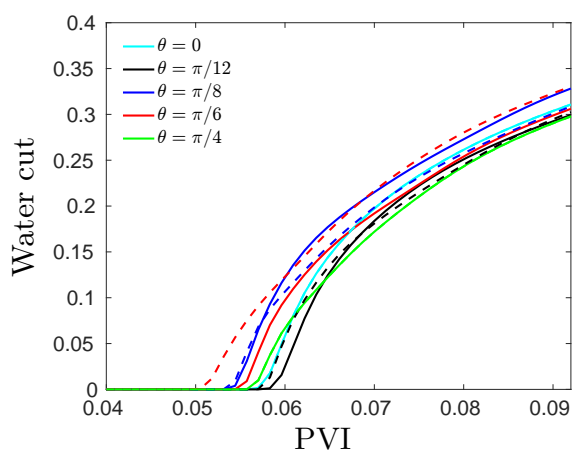

(c) MultiD-PPU

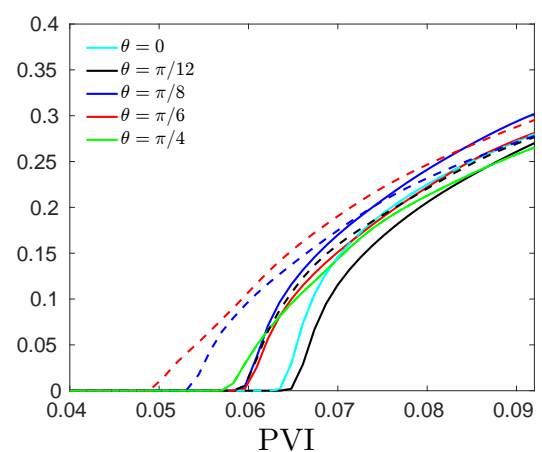

(b) $1 \mathrm{D}-\mathrm{IHU}$

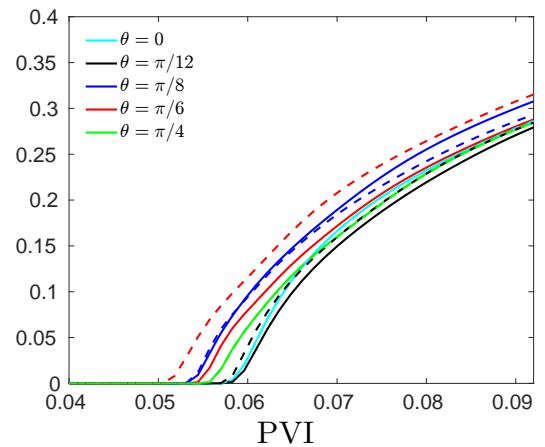

(d) MultiD-IHU

Figure 6: Water cuts at the two producers for different angles $\theta$ at $T=0.092 \mathrm{PVI}$ in the three-well problem with buoyancy. The CFL number of these simulations is 3.4. The solid (respectively, dashed) lines show the water cut for the producer on the left (respectively, on the right).

where $h_{\text {ref }}$ denotes the height of the domain boundaries. Here, $h\left(x_{a}^{\prime}, y_{a}^{\prime}\right)>h\left(x_{b}^{\prime}, y_{b}^{\prime}\right)$ means that $\left(x_{b}^{\prime}, y_{b}^{\prime}\right)$ is deeper than $\left(x_{a}^{\prime}, y_{a}^{\prime}\right)$. With $(60),\left(x_{0}^{\prime}, y_{0}^{\prime}\right)=(0,0)$ is at the top of the domain while the domain boundaries are at the bottom as shown in Fig. 7(b). The domain is initially saturated with the lighter non-wetting phase. A well placed in control volume $\left(x_{0}^{\prime}, y_{0}^{\prime}\right)=(0,0)$ injects a heavy and mobile wetting phase into a domain fully saturated with the light non-wetting phase. We fix the pressure in the control volumes surrounding the disc. The phase properties are the same as in Section 7.1. The gravity number is $N_{G}=12.9$, with countercurrent flow at about $25 \%$ of the interfaces.

The saturation maps after 0.06 PVI are in Fig. 8. They have been obtained with a constant time step of $\Delta t \approx 0.0021 \mathrm{PVI}$ corresponding to a CFL number of 21.1. The saturation pattern is clearly biased by the orientation of the grid with both 1D-PPU and 1D-IHU. This is the case for all angles, but particularly for $\theta=\pi / 12$ and $\theta=\pi / 8$, for which the grid orientation effect is aggravated by the fact that the highpermeability channels are aligned with the main directions of the grid. This nonphysical behavior is significantly attenuated - though not completely eliminated - with the multidimensional schemes. We note that MultiD-PPU and MultiD-IHU yield similar saturation patterns far from the well, even though MultiD-PPU better reduces the numerical biasing caused by the orientation of the grid near the well.

The nonlinear behavior of the four schemes is detailed in Table 2 for two constant time step sizes, namely $\Delta t \approx 0.0021 \mathrm{PVI}$ corresponding to CFL number of 21.1 , and $\Delta t \approx 0.01 \mathrm{PVI}$ for a CFL number of 106.1. The results are consistent with those of Section 7.1. Specifically, MultiD-IHU is the most efficient scheme in terms of Newton iterations, with a total reduction of $10.4 \%$ compared to 1D-PPU for short time steps, and of $13.7 \%$ for larger time steps.

\subsection{Gravity segregation with low-permeability barriers}

Finally, we consider a $x-y$ domain of dimensions $[-75 \mathrm{ft}, 75 \mathrm{ft}] \times[-75 \mathrm{ft}, 75 \mathrm{ft}]$, in which low-permeability layers are placed to slow down the upward migration of the lighter non-wetting phase. The domain is 


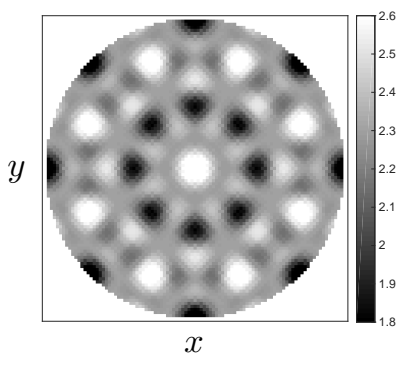

(a)

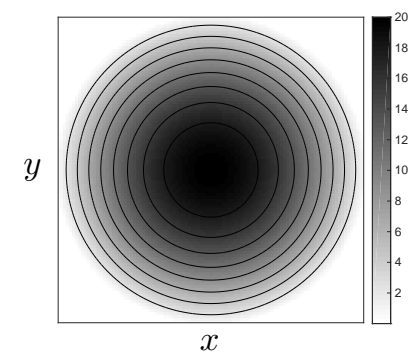

(b)

Figure 7: Logarithm of the absolute permeability field in (a). It is computed with (58) within the disc of radius $r_{0}$ centered in $\left(x_{0}^{\prime}, y_{0}^{\prime}\right)=(0,0)$. The smallest (respectively, largest) permeability is $30.7 \mathrm{mD}$ (respectively, $675 \mathrm{mD}$ ). The height $h_{\text {bump }}$ obtained from $(60)$ is shown in $(b)$. Outside the disc, the absolute permeability is set to $10^{-10} \mathrm{mD}$ (not shown in the figure) and the domain is flat.

\begin{tabular}{ccccccccc}
\hline $\begin{array}{c}\text { Angle } \\
\theta\end{array}$ & 1D-PPU & 1D-IHU & MultiD-PPU & MultiD-IHU & 1D-PPU & 1D-IHU & MultiD-PPU & MultiD-IHU \\
\hline 0 & 167 & 161 & 153 & 146 & 69 & 72 & 62 & 58 \\
$\pi / 12$ & 166 & 161 & 154 & 151 & 69 & 70 & 61 & 59 \\
$\pi / 8$ & 167 & 161 & 154 & 153 & 72 & 71 & 63 & 62 \\
$\pi / 6$ & 167 & 161 & 156 & 151 & 73 & 71 & 62 & 62 \\
$\pi / 4$ & 166 & 161 & 154 & 145 & 68 & 71 & 61 & 62 \\
\hline
\end{tabular}

Table 2: Total number of Newton iterations for different angles at $T=0.06$ PVI in the heterogeneous injection problem with buoyancy.

tilted with an angle of $\pi / 3$ in the direction that is perpendicular to the low-permeability layers. We use a uniform Cartesian grid consisting of $101 \times 101$ control volumes. In the disc of radius $r_{0}=(150-\Delta x) / 2 \mathrm{ft}$ that occupies the center of domain, the absolute permeability is $5 \times 10^{-9} \mathrm{mD}$ in the horizontal layers and $50 \mathrm{mD}$ everywhere else. Outside the disc, the absolute permeability is also equal to $5 \times 10^{-9} \mathrm{mD}$. The absolute permeability field is shown in Fig. 9. We use the same technique as in Sections 7.1 and 7.2 to compute the location of the low-permeability layers as we rotate the grid.

The non-wetting phase is initially saturating the bottom of the domain. Specifically, the initial saturation field is given by

$$
S_{n w}^{\text {init }}(x, y)= \begin{cases}1 & \text { if }-75<y^{\prime}<-45 \text { and } \sqrt{x^{2}+y^{2}} \leq r_{0} \\ 0 & \text { otherwise. }\end{cases}
$$

where the $y^{\prime}$-axis is increasing from $-75 \mathrm{ft}$ to $75 \mathrm{ft}$ when going from bottom to top. The phase densities are given by $\rho_{n w}=32 \mathrm{lb}_{m} . \mathrm{ft}^{-3}$ and $\rho_{w}=64 \mathrm{lb}_{m} . \mathrm{ft}^{-3}$. The constant phase viscosities are chosen to be $\mu_{n w}=2 \mathrm{cP}$ and $\mu_{w}=1 \mathrm{cP}$. Finally, we use Corey-type relative permeabilities such that $k_{r n w}(S)=(1-S)^{2}$ and $k_{r w}(S)=S^{1.5}$.

Figure 10 compares the saturation maps produced by the different schemes after 6000 days once the plume has reached the top of the domain. As in the previous examples, the truly multidimensional schemes drastically reduce the grid orientation effect compared to the schemes based on two-point upwinding. This is particularly visible in the upper part of the domain where the shape of the plume predicted by 1D-PPU and 1D-IHU varies significantly as the grid is rotated and becomes very large for an angle $\theta=\pi / 4$. For this example, the shape of the plume is best preserved for all grid orientations by MultiD-IHU.

The nonlinear iteration count is given in Table 3. For this buoyancy-dominated example, the reduction in the number of nonlinear iterations with MultiD-PPU compared to 1D-PPU is much smaller than in the previous sections. We also note that 1D-IHU exhibits a better nonlinear behavior than MultiD-PPU. MultiD-IHU still achieves a very robust nonlinear behavior that remains insensitive to the orientation of the computational grid. For a CFL number of 8.9 (respectively, 17.8), MultiD-IHU reduces the total 


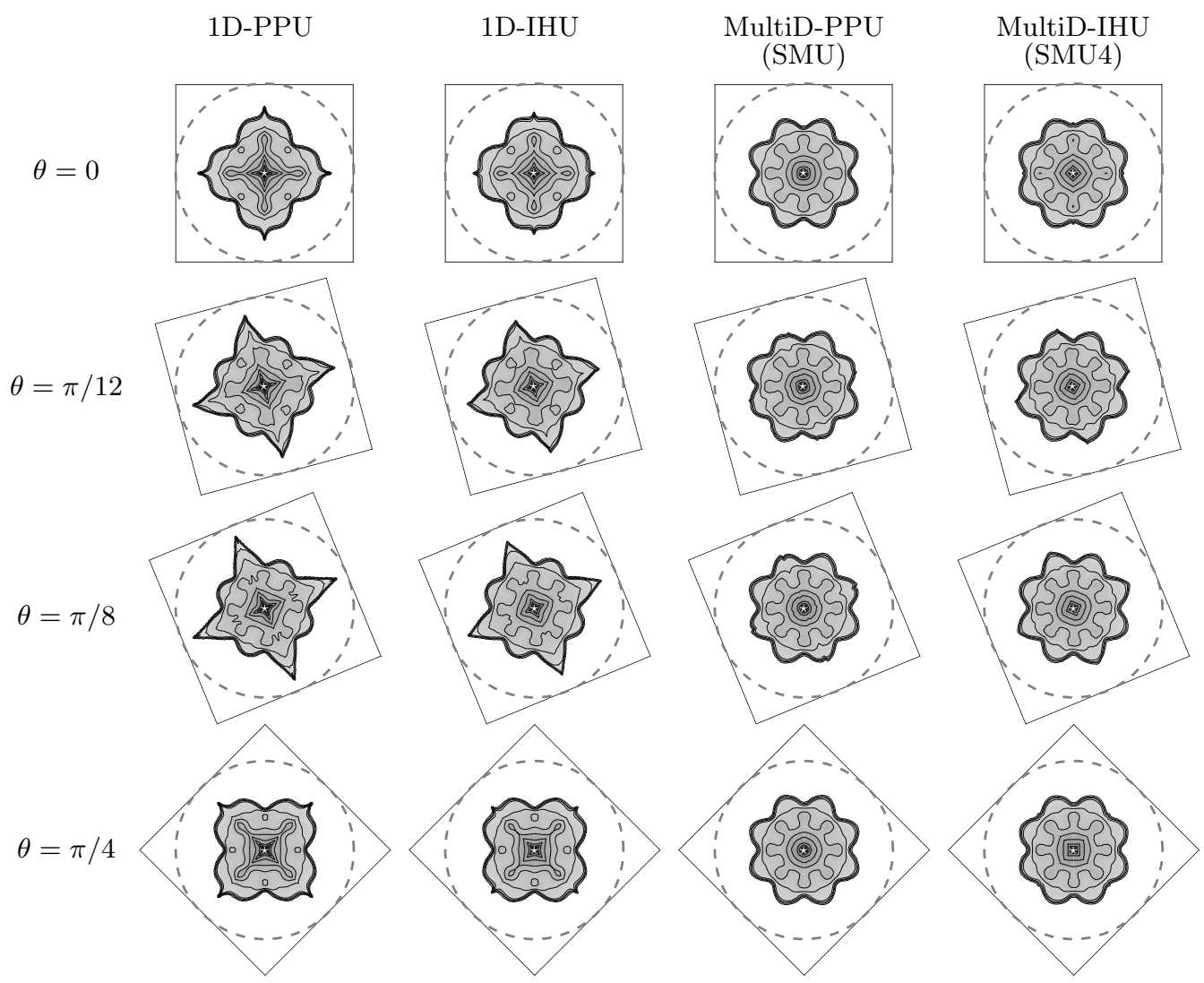

Figure 8: Saturation maps for different values of the angle $\theta$ after 0.06 PVI for the heterogeneous two-phase problem. The CFL number of these simulations is 21.1. The white stars show the location of the injector.

number of nonlinear iterations summed over all cases by $19 \%$ (respectively, 25.8\%) compared to 1D-PPU. It also reduces the number of Newton iterations compared to MultiD-PPU, and performs slightly better than 1D-IHU.

\begin{tabular}{ccccccccc}
\hline $\begin{array}{c}\text { Angle } \\
\theta\end{array}$ & \multicolumn{3}{c}{ Smaller CFL } & \multicolumn{4}{c}{ Larger CFL } \\
\hline 0 & 253 & 238 & 257 & 241 & 199 & 187 & 202 & 182 \\
$\pi / 12$ & 307 & 239 & 292 & 235 & 244 & 171 & 231 & 167 \\
$\pi / 8$ & 290 & 234 & 285 & 231 & 225 & 174 & 222 & 163 \\
$\pi / 6$ & 300 & 236 & 290 & 233 & 226 & 171 & 228 & 158 \\
$\pi / 4$ & 281 & 229 & 277 & 229 & 212 & 167 & 214 & 151 \\
\hline
\end{tabular}

Table 3: Total number of Newton iterations for different angles after 6000 days in the gravity segregation problem with low-permeability layers. There was no time step cut for any of the schemes considered here.

\section{Summary of results and conclusions}

We have proposed a robust fully implicit finite-volume scheme for coupled multiphase flow and transport with buoyancy that is more accurate than commonly used first-order 1D upwinding schemes. Specifically, we have presented a truly multidimensional extension of Implicit Hybrid Upwinding (IHU), referred to as MultiD-IHU, that reduces the numerical biasing due to the orientation of the underlying grid while retaining the monotonicity and robustness of IHU in the presence of competing viscous and buoyancy 


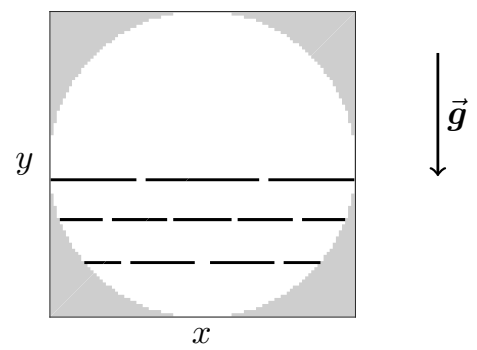

Figure 9: Absolute permeability field with low-permeability layers for an angle $\theta=0$. Outside the disc of radius $r_{0}$ centered in $(x, y)=(0,0)$, the absolute permeability is set to $5 \times 10^{-9} \mathrm{mD}$.

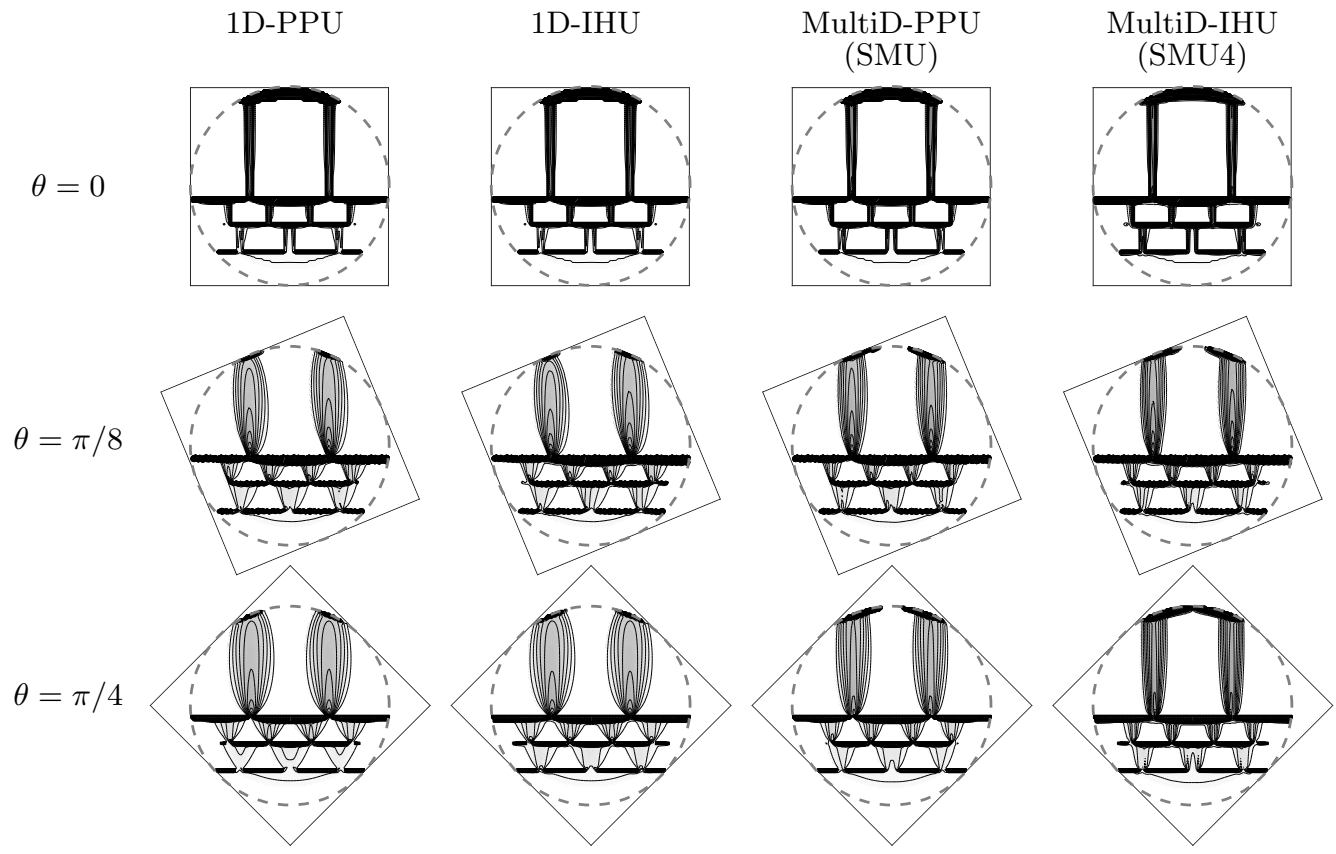

Figure 10: Saturation maps for different angles $\theta$ after 6000 days for the gravity segregation problem with lowpermeability layers. The CFL number of these simulations is 8.9. Outside the dashed circle, the control volumes have an absolute permeability set to $5 \times 10^{-9} \mathrm{mD}$.

forces. This extension builds on the methodology proposed in Kozdon et al. (2011a) for Phase-Potential Upwinding and is based on an extended stencil that adapts to the local flow pattern for the evaluation of the phase mobility ratios.

We have tested the proposed scheme on two-phase flow numerical examples including competing viscous and buoyancy forces and characterized by the presence of countercurrent flow. We have shown that MultiD-IHU reduces the grid orientation effect as well as MultiD-PPU for the two first numerical examples, and leads to a more significant reduction than MultiD-PPU for the gravity segregation case with low-permeability layers. We have also demonstrated that MultiD-IHU retains the robust nonlinear behavior of 1D-IHU, even for large time steps. This is particularly the case for the third numerical example, in which MultiD-IHU reduces the number of nonlinear iterations by up $25.8 \%$ compared to 1D-PPU and by up $25.2 \%$ compared to MultiD-PPU. The computational gain due to the reduction in nonlinear iterations will help offset the added per-iteration cost associated with the larger stencils of multidimensional schemes. Future work includes extending the methodology presented here to a robust, fully implicit high-resolution approach that would tend to a truly accurate scheme. 


\section{Acknowledgments}

The authors thank the SUPRI-B affiliates program at Stanford University, and in particular Prof. Hamdi Tchelepi for his insight and guidance. The first author acknowledges the financial support of Total during his PhD at Stanford University.

\section{Appendix A. Properties of matrices $A$ and $A^{-1} B$}

We study the properties of the matrices $\boldsymbol{A}$ and $\boldsymbol{C} \equiv \boldsymbol{A}^{-1} \boldsymbol{B}$ defined in 290 and 30 . By construction, on each row, $\boldsymbol{A}$ has one positive unit term on the diagonal and one non-positive off-diagonal term. The definition of the SMU4 limiter given in (26) guarantees that

$$
0 \leq \varphi^{S M U_{4}}(r)<1 \quad \forall r \in[0,1] .
$$

Combining A.1 with the definition of the weights in 25, we obtain

$$
0 \leq \bar{\omega}_{k+1 / 2}^{V}<1 \quad \forall k \in\{1, \ldots, 4\} .
$$

Therefore, all the off-diagonal terms in $\boldsymbol{A}$ are strictly smaller than 1, which implies that this matrix is strictly diagonally dominant in the sense of

$$
\left|a_{i i}\right|>\sum_{j \neq i}\left|a_{i j}\right| \quad i \in\{1, \ldots, 4\} .
$$

$\boldsymbol{A}$ is therefore a non-singular M-matrix, and $\boldsymbol{A}^{-1}$ is non-negative. Since $\boldsymbol{B}$ is also non-negative by construction, $\boldsymbol{A}^{-1} \boldsymbol{B}$ is non-negative. To show that for each row of $\boldsymbol{C}$, the sum of the entries is equal to one, i.e., $\sum_{k} c_{i k}=1$ for $i \in\{1, \ldots, 4\}$, we define the vector $\boldsymbol{e}=[1,1,1,1]^{T}$. Then, $\boldsymbol{C e}=\boldsymbol{e}$ is equivalent to $\boldsymbol{A} \boldsymbol{e}=\boldsymbol{B} \boldsymbol{e}$, which follows from the definitions (29) and (30).

\section{Appendix B. Viscous flux monotonicity}

In this section and the next one, we show that the MultiD-IHU scheme is monotone in the sense of 18. . We consider first the monotonicity of the viscous flux by analyzing the sign of the derivatives with respect to saturation of

$$
\bar{V}_{\ell, k+1 / 2}-\bar{V}_{\ell, k-1 / 2}=\bar{\chi}_{\ell, k+1 / 2} \bar{u}_{T, k+1 / 2}-\bar{\chi}_{\ell, k-1 / 2} \bar{u}_{T, k-1 / 2},
$$

for a fixed total velocity field. Following Kozdon et al. (2011a), we consider only three cases since the other cases can be treated by symmetry. We first assume that $\bar{u}_{T, k+1 / 2} \leq 0$ and $\bar{u}_{T, k-1 / 2} \geq 0$. Using (32) and matrix $\boldsymbol{C}=\boldsymbol{A}^{-1} \boldsymbol{B}$, we have

$$
\bar{V}_{\ell, k+1 / 2}-\bar{V}_{\ell, k-1 / 2}=\sum_{j=1}^{4}\left(c_{k j} \bar{u}_{T, k+1 / 2}-c_{(k-1) j} \bar{u}_{T, k-1 / 2}\right) \chi_{\ell, j} .
$$

Using the assumption on the sign of the total velocities and the non-negativity of $\boldsymbol{C} \equiv \boldsymbol{A}^{-1} \boldsymbol{B}$ shown in Appendix A (B.2) gives

$$
\frac{\partial\left(\bar{V}_{\ell, k+1 / 2}-\bar{V}_{\ell, k-1 / 2}\right)}{\partial S_{\ell, j \neq k}}=\left(c_{k j} \bar{u}_{T, k+1 / 2}-c_{(k-1) j} \bar{u}_{T, k-1 / 2}\right) \frac{\partial \chi_{\ell, j}}{\partial S_{\ell, j \neq k}} \leq 0 .
$$

In the second case, we assume that $\bar{u}_{T, k+1 / 2} \geq 0$ and $\bar{u}_{T, k-1 / 2} \leq 0$. Using the definition of the upwinding of the mobility ratio, we can write

$$
\bar{V}_{\ell, k+1 / 2}-\bar{V}_{\ell, k-1 / 2}=\chi_{\ell, k} \bar{u}_{T, k+1 / 2}-\chi_{\ell, k} \bar{u}_{T, k-1 / 2},
$$




\section{Hamon and Mallison}

which yields

$$
\frac{\partial\left(\bar{V}_{\ell, k+1 / 2}-\bar{V}_{\ell, k-1 / 2}\right)}{\partial S_{\ell, j \neq k}}=0 .
$$

The third case is such that $\bar{u}_{T, k+1 / 2} \geq 0$ and $\bar{u}_{T, k-1 / 2} \geq 0$. Using the weighted average of (23), we write

$$
\begin{aligned}
\bar{V}_{\ell, k+1 / 2}-\bar{V}_{\ell, k-1 / 2} & =\left(1-\bar{\omega}_{k+1 / 2}^{V}\right) \chi_{\ell, k} \bar{u}_{T, k+1 / 2}+\left(\bar{\omega}_{k+1 / 2}^{V} \bar{u}_{T, k+1 / 2}-\bar{u}_{T, k-1 / 2}\right) \bar{\chi}_{\ell, k-1 / 2} \\
& =\left(1-\bar{\omega}_{k+1 / 2}^{V}\right) \chi_{\ell, k} \bar{u}_{T, k+1 / 2}+\left(\bar{\omega}_{k+1 / 2}^{V} \bar{u}_{T, k+1 / 2}-\bar{u}_{T, k-1 / 2}\right) \sum_{j=1}^{4} c_{(k-1) j} \chi_{\ell, j} .
\end{aligned}
$$

Taking the derivatives with respect to saturation in $(\mathrm{B.6})$ gives

$$
\frac{\partial\left(\bar{V}_{\ell, k+1 / 2}-\bar{V}_{\ell, k-1 / 2}\right)}{\partial S_{\ell, j \neq k}}=\left(\bar{\omega}_{k+1 / 2}^{V} \bar{u}_{T, k+1 / 2}-\bar{u}_{T, k-1 / 2}\right) c_{(k-1) j} \frac{\partial \chi_{\ell, j}}{\partial S_{\ell, j \neq k}} \leq 0,
$$

since the definition of the limiter guarantees that $\left(\bar{\omega}_{k+1 / 2}^{V} \bar{u}_{T, k+1 / 2}-\bar{u}_{T, k-1 / 2}\right) \leq 0$.

\section{Appendix C. Buoyancy flux monotonicity}

Next, we consider the monotonicity of the buoyancy term by studying the sign of the derivative with respect to saturation of

$$
\bar{G}_{\ell, k+1 / 2}-\bar{G}_{\ell, k-1 / 2}=\bar{T}_{k+1 / 2} \bar{\psi}_{\ell, m, k+1 / 2}\left(\rho_{\ell}-\rho_{m}\right) \overline{\Delta z}_{k+1 / 2}-\bar{T}_{k-1 / 2} \bar{\psi}_{\ell, m, k-1 / 2}\left(\rho_{\ell}-\rho_{m}\right) \overline{\Delta z}_{k-1 / 2} .
$$

We assume that $\rho_{\ell}-\rho_{m}>0$ and $\overline{\Delta z}_{k+1 / 2}>0$. The other cases can be treated analogously and will be omitted for brevity. On a Cartesian grid, we only have to study two configurations. In the first configuration, we assume that $\overline{\Delta z}_{k+3 / 2} \overline{\Delta z}_{k+1 / 2}>0$ and $\overline{\Delta z}_{k+1 / 2} \overline{\Delta z}_{k-1 / 2}<0$, which yields

$$
\begin{aligned}
\bar{G}_{\ell, k+1 / 2} & =\bar{T}_{k+1 / 2}\left(\left(1-\bar{\omega}_{k+1 / 2}^{G}\right) \frac{\lambda_{\ell, k} \lambda_{m, k+1}}{\lambda_{\ell, k}+\lambda_{m, k+1}}+\bar{\omega}_{k+1 / 2}^{G} \frac{\lambda_{\ell, k} \lambda_{m, k+2}}{\lambda_{\ell, k}+\lambda_{m, k+2}}\right)\left(\rho_{\ell}-\rho_{m}\right) \overline{\Delta z}_{k+1 / 2}, \\
\bar{G}_{\ell, k-1 / 2} & =\bar{T}_{k-1 / 2}\left(\left(1-\bar{\omega}_{k-1 / 2}^{G}\right) \frac{\lambda_{\ell, k} \lambda_{m, k-1}}{\lambda_{\ell, k}+\lambda_{m, k-1}}+\bar{\omega}_{k-1 / 2}^{G} \frac{\lambda_{\ell, k} \lambda_{m, k-2}}{\lambda_{\ell, k}+\lambda_{m, k-2}}\right)\left(\rho_{\ell}-\rho_{m}\right) \overline{\Delta z}_{k-1 / 2} .
\end{aligned}
$$

Noting that $m \neq \ell$, the sign of the derivatives of the mobility ratios is given by

$$
\frac{\partial}{\partial S_{\ell, j \neq k}}\left(\frac{\lambda_{\ell, k} \lambda_{m, j}}{\lambda_{\ell, k}+\lambda_{m, j}}\right)=\frac{\lambda_{\ell, k}^{2} \frac{\partial \lambda_{m, j}}{\partial S_{\ell, j \neq k}}}{\left(\lambda_{\ell, k}+\lambda_{m, j}\right)^{2}} \leq 0 .
$$

We now use the assumptions on the densities and the depth to obtain

$$
\frac{\partial\left(\bar{G}_{\ell, k+1 / 2}-\bar{G}_{\ell, k-1 / 2}\right)}{\partial S_{\ell, j \neq k}} \leq 0 .
$$

In the second configuration, we assume that $\overline{\Delta z}_{k+3 / 2} \overline{\Delta z}_{k+1 / 2}<0$ and $\overline{\Delta z}_{k+1 / 2} \overline{\Delta z}_{k-1 / 2}>0$. This case yields

$$
\begin{aligned}
\bar{G}_{\ell, k+1 / 2} & =\bar{T}_{k+1 / 2}\left(\left(1-\bar{\omega}_{k+1 / 2}^{G}\right) \frac{\lambda_{\ell, k} \lambda_{m, k+1}}{\lambda_{\ell, k}+\lambda_{m, k+1}}+\bar{\omega}_{k+1 / 2}^{G} \frac{\lambda_{\ell, k-1} \lambda_{m, k+1}}{\lambda_{\ell, k-1}+\lambda_{m, k+1}}\right)\left(\rho_{\ell}-\rho_{m}\right) \overline{\Delta z}_{k+1 / 2}, \\
\bar{G}_{\ell, k-1 / 2} & =\bar{T}_{k-1 / 2}\left(\left(1-\bar{\omega}_{k-1 / 2}^{G}\right) \frac{\lambda_{\ell, k-1} \lambda_{m, k}}{\lambda_{\ell, k-1}+\lambda_{m, k}}+\bar{\omega}_{k-1 / 2}^{G} \frac{\lambda_{\ell, k-1} \lambda_{m, k+1}}{\lambda_{\ell, k-1}+\lambda_{m, k+1}}\right)\left(\rho_{\ell}-\rho_{m}\right) \overline{\Delta z}_{k-1 / 2} .
\end{aligned}
$$


Using these expressions, we obtain

$$
\begin{aligned}
\bar{G}_{\ell, k+1 / 2}-\bar{G}_{\ell, k-1 / 2} & =\left(\bar{T}_{k+1 / 2}\left(1-\bar{\omega}_{k+1 / 2}^{G}\right) \frac{\lambda_{\ell, k} \lambda_{m, k+1}}{\lambda_{\ell, k}+\lambda_{m, k+1}} \overline{\Delta z}_{k+1 / 2}\right. \\
& \left.-\bar{T}_{k-1 / 2}\left(1-\bar{\omega}_{k-1 / 2}^{G}\right) \frac{\lambda_{\ell, k-1} \lambda_{m, k}}{\lambda_{\ell, k-1}+\lambda_{m, k}} \overline{\Delta z}_{k-1 / 2}\right)\left(\rho_{\ell}-\rho_{m}\right) \\
& +\left(\bar{T}_{k+1 / 2} \bar{\omega}_{k+1 / 2}^{G} \overline{\Delta z}_{k+1 / 2}-\bar{T}_{k-1 / 2} \bar{\omega}_{k-1 / 2}^{G} \overline{\Delta z}_{k-1 / 2}\right) \frac{\lambda_{\ell, k-1} \lambda_{m, k+1}}{\lambda_{\ell, k-1}+\lambda_{m, k+1}}\left(\rho_{\ell}-\rho_{m}\right) .
\end{aligned}
$$

Equation 27) guarantees that $\bar{T}_{k+1 / 2} \bar{\omega}_{k+1 / 2}^{G} \overline{\Delta z}_{k+1 / 2}-\bar{T}_{k-1 / 2} \bar{\omega}_{k-1 / 2}^{G} \overline{\Delta z}_{k-1 / 2}=0$ whenever $\overline{\Delta z}_{k+3 / 2} \overline{\Delta z}_{k+1 / 2}<$ 0 and $\overline{\Delta z}_{k+1 / 2} \overline{\Delta z}_{k-1 / 2}>0$. Therefore the term in the third line of C.8 cancels and we can use the sign of the derivatives of the mobility ratios to obtain the result

$$
\frac{\partial\left(\bar{G}_{\ell, k+1 / 2}-\bar{G}_{\ell, k-1 / 2}\right)}{\partial S_{\ell, j \neq k}} \leq 0 .
$$

\section{References}

Aavatsmark, I. (2002). An introduction to multipoint flux approximations for quadrilateral grids. Computational Geosciences, 6(3-4):405-432.

Aavatsmark, I., Barkve, T., and Mannseth, T. (1998). Control-volume discretization methods for 3D quadrilateral grids in inhomogeneous, anisotropic reservoirs. SPE Journal, 3(2):146-154.

Arbogast, T. and Huang, C.-S. (2006). A fully mass and volume conserving implementation of a characteristic method for transport problems. SIAM Journal on Scientific Computing, 28(6):2001-2022.

Arbogast, T., Huang, C.-S., and Zhao, X. (2019). Von neumann stable, implicit, high order, finite volume WENO schemes. In SPE Reservoir Simulation Conference. Society of Petroleum Engineers.

Aziz, K. and Settari, A. (1979). Petroleum reservoir simulation, volume 476. Applied Science Publishers London.

Berger, M. J., Helzel, C., and LeVeque, R. J. (2003). H-box methods for the approximation of hyperbolic conservation laws on irregular grids. SIAM Journal on Numerical Analysis, 41(3):893-918.

Blunt, M. and Rubin, B. (1992). Implicit flux limiting schemes for petroleum reservoir simulation. Journal of Computational Physics, 102(1):194-210.

Brand, C. W., Heinemann, J. E., and Aziz, K. (1991). The grid orientation effect in reservoir simulation. In SPE Symposium on Reservoir Simulation. Society of Petroleum Engineers.

Brenier, Y. and Jaffré, J. (1991). Upstream differencing for multiphase flow in reservoir simulation. SIAM Journal on Numerical Analysis, 28(3):685-696.

Brenner, K., Cancès, C., and Hilhorst, D. (2013). Finite volume approximation for an immiscible two-phase flow in porous media with discontinuous capillary pressure. Computational Geosciences, 17(3):573-597.

Chen, W. H., Durlofsky, L. J., Engquist, B., and Osher, S. (1993). Minimization of grid orientation effects through use of higher order finite difference methods. SPE Advanced Technology Series, 1(2):43-52.

Colella, P. (1990). Multidimensional upwind methods for hyperbolic conservation laws. Journal of Computational Physics, 87(1):171-200.

Corey, A. T. (1954). The interrelation between gas and oil relative permeabilities. Producers Monthly, 19(1):38-41.

Deuflhard, P. (2011). Newton methods for nonlinear problems: affine invariance and adaptive algorithms, volume 35. Springer.

Edwards, M. G. (2011). Multi-dimensional wave-oriented upwind schemes with reduced cross-wind diffusion for flow in porous media. International Journal for Numerical Methods in Fluids, 67(1):33-57.

Edwards, M. G. and Rogers, C. F. (1998). Finite volume discretization with imposed flux continuity for the general tensor pressure equation. Computational Geosciences, 2(4):259-290.

Eymard, R., Gallouët, T., and Joly, P. (1989). Hybrid finite element techniques for oil recovery simulation. Computer Methods in Applied Mechanics and Engineering, 74(1):83-98. 
Eymard, R., Guichard, C., and Masson, R. (2012). Grid orientation effect in coupled finite volume schemes. IMA Journal of Numerical Analysis, 33(2):582-608.

Hamon, F. P., Mallison, B. T., and Tchelepi, H. A. (2016). Implicit hybrid upwind scheme for coupled multiphase flow and transport with buoyancy. Computer Methods in Applied Mechanics and Engineering, 311:599-624.

Hamon, F. P., Mallison, B. T., and Tchelepi, H. A. (2018). Implicit hybrid upwinding for two-phase flow in heterogeneous porous media with buoyancy and capillarity. Computer Methods in Applied Mechanics and Engineering, 331:701-727.

Hamon, F. P. and Tchelepi, H. A. (2016). Analysis of hybrid upwinding for fully implicit simulation of three-phase flow with gravity. SIAM Journal on Numerical Analysis, 54(3):1682-1712.

Hurtado, F. S. V., Maliska, C. R., Carvalho da Silva, A. F., and Cordazzo, J. (2007). A quadrilateral element-based finite-volume formulation for the simulation of complex reservoirs. In Latin American 83 Caribbean Petroleum Engineering Conference. Society of Petroleum Engineers.

Keilegavlen, E., Kozdon, J. E., and Mallison, B. T. (2012). Multidimensional upstream weighting for multiphase transport on general grids. Computational Geosciences, 16(4):1021-1042.

Koren, B. (1991). Low-diffusion rotated upwind schemes, multigrid and defect correction for steady, multi-dimensional Euler flows. In Multigrid Methods III, pages 265-276. Springer.

Kozdon, J. E., Mallison, B. T., and Gerritsen, M. G. (2009). Robust multi-D transport schemes with reduced grid orientation effects. Transport in Porous Media, 78(1):47-75.

Kozdon, J. E., Mallison, B. T., and Gerritsen, M. G. (2011a). Multidimensional upstream weighting for multiphase transport in porous media. Computational Geosciences, 15(3):399-419.

Kozdon, J. E., Mallison, B. T., Gerritsen, M. G., and Chen, W. H. (2011b). Multidimensional upwinding for multiphase transport in porous media. SPE Journal, 16(2):263-272.

Lamine, S. and Edwards, M. G. (2010). Higher order multidimensional upwind convection schemes for flow in porous media on structured and unstructured quadrilateral grids. SIAM Journal on Scientific Computing, 32(3):1119-1139.

Lamine, S. and Edwards, M. G. (2013). Higher order cell-based multidimensional upwind schemes for flow in porous media on unstructured grids. Computer Methods in Applied Mechanics and Engineering, 259:103-122.

Lamine, S. and Edwards, M. G. (2015). Multidimensional upwind schemes and higher resolution methods for three-component two-phase systems including gravity driven flow in porous media on unstructured grids. Computer Methods in Applied Mechanics and Engineering, 292:171-194.

Lee, S. H. and Efendiev, Y. (2016). $\mathrm{C}^{1}$-continuous relative permeability and hybrid upwind discretization of three phase flow in porous media. Advances in Water Resources, 96:209-224.

Lee, S. H. and Efendiev, Y. (2018). Hybrid discretization of multi-phase flow in porous media in the presence of viscous, gravitational, and capillary forces. Computational Geosciences, pages 1-19.

Lee, S. H., Efendiev, Y., and Tchelepi, H. A. (2015). Hybrid upwind discretization of nonlinear two-phase flow with gravity. Advances in Water Resources, 82:27-38.

LeVeque, R. J. (1997). Wave propagation algorithms for multidimensional hyperbolic systems. Journal of Computational Physics, 131(2):327-353.

Li, B. and Tchelepi, H. A. (2015). Nonlinear analysis of multiphase transport in porous media in the presence of viscous, buoyancy, and capillary forces. Journal of Computational Physics, 297:104-131.

Mallison, B., Gerritsen, M., Jessen, K., and Orr, F. (2005). High order upwind schemes for two-phase, multicomponent flow. SPE Journal, 10(03):297-311.

Moncorgé, A., Møyner, O., Tchelepi, H. A., and Jenny, P. (2019). Consistent upwinding for sequential fully implicit multiscale compositional simulation. Computational Geosciences, pages 1-18.

Mykkeltvedt, T. S., Raynaud, X., and Lie, K.-A. (2017). Fully implicit higher-order schemes applied to polymer flooding. Computational Geosciences, 21(5-6):1245-1266.

Peaceman, D. W. (1977). Fundamentals of numerical reservoir simulation. Elsevier.

Pruess, K. and Bodvarsson, F. S. (1983). A seven-point finite difference method for improved grid orientation performance in pattern steamfloods. In SPE Reservoir Simulation Symposium. Society of Petroleum Engineers.

Roe, P. L. and Sidilkover, D. (1992). Optimum positive linear schemes for advection in two and three dimensions. SIAM Journal on Numerical Analysis, 29(6):1542-1568. 
Sammon, P. H. (1988). An analysis of upstream differencing. SPE Reservoir Engineering, 3(3):1053-1056. Schneider, G. E. and Raw, M. J. (1986). A skewed, positive influence coefficient upwinding procedure for control-volume-based finite-element convection-diffusion computation. Numerical Heat Transfer, Part A: Applications, 9(1):1-26.

Shubin, G. R. and Bell, J. B. (1984). An analysis of the grid orientation effect in numerical simulation of miscible displacement. Computer Methods in Applied Mechanics and Engineering, 47(1-2):47-71.

Souza, M. R. A., Contreras, F. R. L., Lyra, P. R. M., and Carvalho, D. K. E. (2018). A higher-resolution flow-oriented scheme with an adaptive correction strategy for distorted meshes coupled with a robust MPFA-D method for the numerical simulation of two-phase flow in heterogeneous and anisotropic petroleum reservoirs. SPE Journal.

Trangenstein, J. A. and Bell, J. B. (1989a). Mathematical structure of compositional reservoir simulation. SIAM Journal on Scientific and Statistical Computing, 10(5):817-845.

Trangenstein, J. A. and Bell, J. B. (1989b). Mathematical structure of the Black-Oil model for petroleum reservoir simulation. SIAM Journal on Applied Mathematics, 49(3):749-783.

Wang, X. and Tchelepi, H. A. (2013). Trust-region based solver for nonlinear transport in heterogeneous porous media. Journal of Computational Physics, 253:114-137.

Xie, Y. and Edwards, M. G. (2019). Unstructured CVD-MPFA reduced-dimensional DFM models for two-phase flow, coupled with higher resolution hybrid upwind methods. In SPE Reservoir Simulation Conference. Society of Petroleum Engineers.

Yanosik, J. L. and McCracken, T. A. (1979). A nine-point, finite-difference reservoir simulator for realistic prediction of adverse mobility ratio displacements. Society of Petroleum Engineers Journal, 19(4):253262.

Zhou, Y., Tchelepi, H. A., and Mallison, B. T. (2011). Automatic differentiation framework for compositional simulation on unstructured grids with multi-point discretization schemes. In SPE Reservoir Simulation Symposium. Society of Petroleum Engineers. 